\title{
On Spawning and Combination of Extended/Group Targets Modeled with Random Matrices
}

Karl Granström and Umut Orguner

\section{Linköping University Post Print}

N.B.: When citing this work, cite the original article.

C2013 IEEE. Personal use of this material is permitted. However, permission to reprint/republish this material for advertising or promotional purposes or for creating new collective works for resale or redistribution to servers or lists, or to reuse any copyrighted component of this work in other works must be obtained from the IEEE.

Karl Granström and Umut Orguner, On Spawning and Combination of Extended/Group Targets Modeled with Random Matrices, 2013, IEEE Transactions on Signal Processing, (61), 3, .

http://dx.doi.org/10.1109/TSP.2012.2230171

Postprint available at: Linköping University Electronic Press

http://urn.kb.se/resolve?urn=urn:nbn:se:liu:diva-82005 


\title{
On Spawning and Combination of Extended/Group Targets Modeled with Random Matrices
}

\author{
Karl Granström, Student Member, IEEE, and Umut Orguner, Member, IEEE
}

\begin{abstract}
In extended/group target tracking, where the extensions of the targets are estimated, target spawning and combination events might have significant implications on the extensions. This paper investigates target spawning and combination events for the case that the target extensions are modeled in a random matrix framework. The paper proposes functions that should be provided by the tracking filter in such a scenario. The results, which are obtained by a gamma Gaussian inverse Wishart implementation of an extended target probability hypothesis density filter, confirms that the proposed functions improve the performance of the tracking filter for spawning and combination events.
\end{abstract}

Index Terms-Extended target, random matrix, KullbackLeibler divergence, target spawning, target combination.

\section{INTRODUCTION}

Multiple target tracking can be defined as the processing of multiple measurements obtained from multiple targets in order to maintain estimates of the targets' current states, see e.g. [1]. In this context, a point target is defined as a target which is assumed to give rise to at most one measurement per time step, and an extended target is defined as a target that potentially gives rise to more than one measurement per time step. Closely related to extended target is group target, defined as a cluster of point targets which can not be tracked individually, but has to be treated as a single object.

In a target tracking scenario, multiple targets may maneuver such that they become spatially close and cannot be resolved, i.e. they appear at the sensor as one $\operatorname{target}^{1}$ and must be treated as such. Conversely, the individual targets in a group of unresolved targets may maneuver such that they become resolved, i.e. they no longer have to be treated as a group. In this paper, we refer to the former as the target combination problem, and to the latter as the target spawning problem.

Target spawning, also referred to as splitting targets, is the event that a new target appears very close to an existing target, or the event that a single target separates into two, or more, targets. Spawning occurs e.g. when a platform launches another platform, or an unresolved group of targets resolve into multiple closely spaced targets, see e.g. [2], [3]. An interactive

Karl Granström is with the Department of Electrical Engineering, Division of Automatic Control, Linköping University, Linköping, SE-581 83, Sweden, e-mail: karleisy.liu.se.

Umut Orguner is with the Department of Electrical and Electronics Engineering, Middle East Technical University, 06531, Ankara, Turkey, e-mail: umut deee.metu.edu.tr.

Copyright (c) 2012 IEEE. Personal use of this material is permitted. However, permission to use this material for any other purposes must be obtained from the IEEE by sending a request to pubs-permissionseieee.org.

${ }^{1}$ Sometimes called group target. multiple model joint probabilistic data association filter for tracking a single point target that spawns one point target is given in [2, Chapter 4].

Target combination, also referred to as target merge, is the event that multiple single targets form a group of targets. In certain scenarios target combination can efficiently be seen, and modeled, as target death ${ }^{2}$. In other scenarios it may be computationally efficient to combine resolved single targets into a group, see e.g. [4].

While tracking point targets, target spawning and combination events can be handled by additional target births around the main target and spontaneous target deaths, respectively, in the tracking filter. On the other hand, in extended/group target tracking where the target/group size should also be estimated by the tracker, a target spawning event might potentially cause a reduction in the size/extent of the main target. Likewise, in the case of target combination, the size of the combined target logically can become the sum of the sizes of the individual targets. This interesting phenomenon that can be observed in extended and group target tracking, but not in conventional point target tracking, has to be modeled and taken care of in the tracking filter.

In this paper we consider combination and spawning for extended targets. An extended target's size and shape can be modeled in different ways, see e.g. [5]-[11], here we use Koch's random matrix model [12]. We limit the discussion to considering combination of two targets, and spawning of one new target, or equivalently splitting into two targets.

To the best of the authors' knowledge, there is no previous work on extended/group target combination, and the only work that mentions extended/group target spawning is [13]. The work [13], which also uses the random matrix model [12], proposes a spawning model that corresponds to a spawned target whose state's expected value is identical to the expected value of the state of the target from which it spawned. This includes the spawned target's extension, which also keeps the expected value of the original target's extension.

This very simple model cannot be expected to be valid in all scenarios, especially not when the original target extension is large and the spawned target's extension is small, which is a quite common case. The spawning model presented in this paper uses a multiple hypothesis structure that considers reasonable alternatives about the spawned target. The spawning model in [13] has a single hypothesis in which the expected kinematic and extension states are equal to the original target. Therefore the model in [13] can be considered to be a special

\footnotetext{
${ }^{2}$ When a target disappears from sensor view.
} 
case of the presented model.

The rest of the paper is organized as follows. In Section II we present the extended target tracking framework, and give a problem formulation. Section III contains results on the approximation of probability density functions, in the form of four theorems that will be used in the subsequent parts of the paper. In Sections IV and V we present the proposed combination and spawning methodologies, respectively, for the two target case. A discussion about how the presented methodologies could be used if another extension model is used is presented in Section VI. A simulation study is presented in Section VIII, using an example multiple extended target tracking filter which is briefly described in Section VII. The paper is finished with concluding remarks in Section IX.

\section{EXTENDED TARGET FRAMEWORK AND PROBLEM FORMULATION}

We use the following notation:

- $\mathbb{R}^{n}$ is the set of real column vectors of length $n, \mathbb{S}_{++}^{n}$ is the set of symmetric positive definite $n \times n$ matrices, and $\mathbb{S}_{+}^{n}$ is the set of symmetric positive semi-definite $n \times n$ matrices.

- $\mathcal{G} \mathcal{A M}(\gamma ; \alpha, \beta)$ denotes a gamma probability density function (pdf) defined over the scalar $\gamma>0$ with scalar shape parameter $\alpha>0$ and scalar inverse scale parameter $\beta>0$,

$$
\mathcal{G} \mathcal{A M}(\gamma ; \alpha, \beta)=\frac{\beta^{\alpha}}{\Gamma(\alpha)} \gamma^{\alpha-1} e^{-\beta \gamma}
$$

where $\Gamma(\cdot)$ is the gamma function.

- $\mathcal{N}(\mathbf{x} ; \mathbf{m}, P)$ denotes a multi-variate Gaussian pdf defined over the vector $\mathbf{x} \in \mathbb{R}^{n_{x}}$ with mean vector $\mathbf{m} \in$ $\mathbb{R}^{n_{x}}$, and covariance matrix $P \in \mathbb{S}_{+}^{n_{x}}$,

$$
\mathcal{N}(\mathbf{x} ; \mathbf{m}, P)=\frac{e^{-\frac{1}{2}(\mathbf{x}-\mathbf{m})^{\mathrm{T}} P^{-1}(\mathbf{x}-\mathbf{m})}}{(2 \pi)^{\frac{n_{x}}{2}}|P|^{\frac{1}{2}}} .
$$

where $|\cdot|$ is the matrix determinant function.

- $\mathcal{I}_{d}(X ; v, V)$ denotes an inverse Wishart pdf defined over the matrix $X \in \mathbb{S}_{++}^{d}$ with scalar degrees of freedom $v>2 d$ and parameter matrix $V \in \mathbb{S}_{++}^{d},[14$, Definition 3.4.1]

$\mathcal{I W}_{d}(X ; v, V)=\frac{2^{-\frac{v-d-1}{2}}|V|^{\frac{v-d-1}{2}}}{\Gamma_{d}\left(\frac{v-d-1}{2}\right)|X|^{\frac{v}{2}}} \operatorname{etr}\left(-\frac{1}{2} X^{-1} V\right)$,

where $\operatorname{etr}(\cdot)=\exp (\operatorname{Tr}(\cdot))$ is exponential of the matrix trace, and $\Gamma_{d}(\cdot)$ is the multivariate gamma function. The multivariate gamma function can be expressed as a product of ordinary gamma functions, see (80) in Appendix D.

- $\mathcal{W}_{d}(X ; w, W)$ denotes a Wishart pdf defined over the matrix $X \in \mathbb{S}_{++}^{d}$ with scalar degrees of freedom $w \geq d$ and parameter matrix $W \in \mathbb{S}_{++}^{d}$, [14, Definition 3.2.1]

$$
\mathcal{W}_{d}(X ; w, W)=\frac{2^{-\frac{w d}{2}}|X|^{\frac{w-d-1}{2}}}{\Gamma_{d}\left(\frac{w}{2}\right)|W|^{\frac{n}{2}}} \operatorname{etr}\left(-\frac{1}{2} W^{-1} X\right) \text {. }
$$

- $\mathcal{B E}(\bar{\gamma} ; a, b)$ denotes a beta pdf defined over the scalar $0<\bar{\gamma}<1$ with scalar shape parameters $a>0$ and $b>0$,

$$
\mathcal{B E}(\bar{\gamma} ; a, b)=\frac{\Gamma(a+b)}{\Gamma(a) \Gamma(b)} \bar{\gamma}^{a-1}(1-\bar{\gamma})^{b-1} .
$$

Let $\xi_{k}$ be the extended target state at time $t_{k}$. In this paper we define the extended target state as the combination of a scalar Poisson rate $\gamma_{k}>0$, a kinematical state vector $\mathbf{x}_{k} \in$ $\mathbb{R}^{n_{x}}$ and an extension state matrix $X_{k} \in \mathbb{S}_{++}^{d}$, i.e. the extended target state is a triple $\xi_{k} \triangleq\left(\gamma_{k}, \mathbf{x}_{k}, X_{k}\right)$. The kinematical state $\mathbf{x}_{k}$ contains states related to target kinematics, such as position, velocity and heading, while the extension state $X_{k}$ is a random matrix representing the size and shape of the target. At time $t_{k}$, each extended target generates a set of sensor measurements

$$
\mathbf{Z}_{k}=\left\{\mathbf{z}_{k}^{(j)}\right\}_{j=1}^{N_{z, k}},
$$

where the measurement noise covariance is related to the extension $X_{k}$. In this paper we use the following measurement model from [12],

$$
p\left(\mathbf{z}_{k}^{(j)} \mid \mathbf{x}_{k}, X_{k}\right)=\mathcal{N}\left(\mathbf{z}_{k}^{(j)} ; H_{k} \mathbf{x}_{k}, X_{k}\right) .
$$

The measurement set cardinality $N_{z, k}$ is a random draw from a Poisson distribution whose unknown rate is $\gamma_{k}$.

Let $\mathbf{Z}^{k}=\left\{\mathbf{Z}_{1}, \ldots, \mathbf{Z}_{k}\right\}$ denote all measurement sets up to and including time $t_{k}$. The state estimate, conditioned on $\mathbf{Z}^{k}$, is assumed to be gamma Gaussian inverse Wishart (GGIW) distributed,

$$
\begin{aligned}
p\left(\xi_{k} \mid \mathbf{Z}^{k}\right)= & p\left(\gamma_{k} \mid \mathbf{Z}^{k}\right) p\left(\mathbf{x}_{k} \mid X_{k}, \mathbf{Z}^{k}\right) p\left(X_{k} \mid \mathbf{Z}^{k}\right) \\
= & \mathcal{G} \mathcal{A} \mathcal{M}\left(\gamma_{k} ; \alpha_{k \mid k}, \beta_{k \mid k}\right) \\
& \times \mathcal{N}\left(\mathbf{x}_{k} ; \mathbf{m}_{k \mid k}, P_{k \mid k} \otimes X_{k}\right) \\
& \times \mathcal{I} \mathcal{W}_{d}\left(X_{k} ; v_{k \mid k}, V_{k \mid k}\right) \\
= & \mathcal{G} \mathcal{G} \mathcal{I}\left(\xi_{k} ; \zeta_{k \mid k}\right),
\end{aligned}
$$

where $A \otimes B$ is the Kronecker product between matrices $A$ and $B$, and $\zeta_{k \mid k}=\left(\alpha_{k \mid k}, \beta_{k \mid k}, \mathbf{m}_{k \mid k}, P_{k \mid k}, v_{k \mid k}, V_{k \mid k}\right)$ is the set of GGIW density parameters. The Gaussian covariance is $\left(P_{k \mid k} \otimes X_{k}\right) \in \mathbb{S}_{+}^{n_{x}}$, where $P_{k \mid k} \in \mathbb{S}_{+}^{s}$, and we thus have $n_{x}=d s$ (refer to [12] for further details).

Decomposing the target kinematics and extension into a Gaussian distributed random vector $\mathbf{x}_{k}$ and an inverse Wishart distributed random matrix $X_{k}$ was proposed by Koch [12], see also [15]. As in [16], the Poisson rate is modeled as gamma distributed because the gamma distribution is the conjugate prior for the Poisson rate, see e.g. [17].

The model (8) assumes the Poisson rate $\gamma_{k}$ to be conditionally independent of $\mathbf{x}_{k}$ and $X_{k}$. In many applications the number of measurements depends on the distance between the sensor and the target, i.e. on the kinematical position, and also depends on the size of the target, i.e. on the size of the extension. This assumption neglects such dependencies, however the probability density over the number of measurements, conditioned on the target kinematics and extension, is unknown in many applications, and we believe that this assumption is valid in many cases. Furthermore, the assumption also facilitates further analysis. Modeling the extension 
as a random matrix limits the extended targets to be shaped as ellipses, however the ellipse shape is applicable to many real scenarios in which the target and sensor geometry is such that the target measurements resemble a cluster of detections, rather than a geometric structure (or for that matter a single detection). Finally, it is also assumed that multiple targets evolve independently over time, and generate measurements independently. This assumption is typical in multiple target tracking, see e.g. [1].

The first problem considered in this paper is two target combination, i.e. finding the GGIW distribution that corresponds to a group of two independent GGIW distributed extended target estimates. The second problem is target spawning, i.e. finding two GGIW distributions that corresponds to either the splitting of a GGIW distributed extended target estimate, or the appearance of a new GGIW distributed extended target estimate next to an existing estimate.

\section{PRELIMINARY RESUltS ON PROBABILITY DENSITY APPROXIMATIONS}

In this section we present four probability density approximations, that are all needed in the derivation of the main result. The true densities are approximated by analytical minimization of the Kullback-Leibler divergence (KL-div) [18], defined for two pdfs $p(x)$ and $q(x)$ as

$$
\mathrm{KL}(p(x) \| q(x))=\int p(x) \log (p(x) / q(x)) \mathrm{d} x .
$$

Note that, when it comes to approximating distributions in a maximum likelihood sense, the KL-div is considered the optimal difference measure [19]-[21].

\section{A. Approximating the distribution of functions of gamma distributed random variables}

Let $\gamma_{1}$ and $\gamma_{2}$ be two gamma distributed random variables,

$$
\begin{aligned}
& p\left(\gamma_{1}\right)=\mathcal{G} \mathcal{A M}\left(\gamma_{1} ; \alpha_{1}, \beta_{1}\right), \\
& p\left(\gamma_{2}\right)=\mathcal{G} \mathcal{A M}\left(\gamma_{2} ; \alpha_{2}, \beta_{2}\right) .
\end{aligned}
$$

It is here of interest to approximate the distributions over $\gamma=\gamma_{1}+\gamma_{2}$ and $\bar{\gamma}_{1}=\frac{\gamma_{1}}{\gamma_{1}+\gamma_{2}}$. There are some convenient properties for these quantities which we summarize in (64) and (65) in Appendix A. However, for these properties to hold, the inverse scale parameters $\beta_{1}$ and $\beta_{2}$ must be equal. We investigate below the general case where $\beta_{1}$ and $\beta_{2}$ need not be equal.

1) Approximate distribution of $\gamma$ :

Theorem 1: Let $\gamma_{1}$ and $\gamma_{2}$ be distributed as in (10), and let $p(\gamma)$ be the true distribution of $\gamma=\gamma_{1}+\gamma_{2}$. Let $q(\gamma)=\mathcal{G A M}(\gamma ; \alpha, \beta)$ be the gamma distribution, among all gamma distributions, that minimizes the KL-div between $p(\gamma)$ and $q(\gamma)$,

$$
q(\gamma)=\underset{q(\cdot) \in \mathcal{G} \mathcal{A M}(\cdot)}{\arg \min } \operatorname{KL}(p(\gamma) \| q(\gamma)) .
$$

Then the shape parameter $\alpha$ is the solution to

$$
\log (\alpha)-\psi_{0}(\alpha)+\mathrm{E}_{p}[\log (\gamma)]-\log \left(\mathrm{E}_{p}[\gamma]\right)=0,
$$

where $\psi_{0}(\cdot)$ is the digamma function (a.k.a. the polygamma function of order 0 ), and the inverse scale parameter $\beta$ is given by

$$
\beta=\frac{\alpha}{\mathrm{E}_{p}[\gamma]} .
$$

Proof: Given in Appendix E.

Remark: The expressions for the shape parameter (12) and the inverse scale parameter (13) correspond to equating the expected values of $\log (\gamma)$ and $\gamma$, respectively, under both distributions,

$$
\begin{aligned}
\mathrm{E}_{p}[\log (\gamma)] & =\mathrm{E}_{q}[\log (\gamma)], \\
\mathrm{E}_{p}[\gamma] & =\mathrm{E}_{q}[\gamma] .
\end{aligned}
$$

2) Approximate distribution of $\bar{\gamma}_{1}$ :

Theorem 2: Let $\gamma_{1}$ and $\gamma_{2}$ be distributed as in (10), and let $p\left(\bar{\gamma}_{1}\right)$ be the true distribution of $\bar{\gamma}_{1}=\frac{\gamma_{1}}{\gamma_{1}+\gamma_{2}}$. Let $q\left(\bar{\gamma}_{1}\right)=\mathcal{B E}\left(\bar{\gamma}_{1} ; a, b\right)$ be the beta distribution, among all beta distributions, that minimizes the KL-div between $p\left(\bar{\gamma}_{1}\right)$ and $q\left(\bar{\gamma}_{1}\right)$,

$$
q\left(\bar{\gamma}_{1}\right)=\underset{q(\cdot) \in \mathcal{B E}(\cdot)}{\arg \min } \operatorname{KL}\left(p\left(\bar{\gamma}_{1}\right) \| q\left(\bar{\gamma}_{1}\right)\right) .
$$

Then the shape parameters $a$ and $b$ are the solution to the system of equations

$$
\left\{\begin{array}{l}
\psi_{0}(a+b)-\psi_{0}(a)+\mathrm{E}_{p}\left[\log \left(\bar{\gamma}_{1}\right)\right]=0 \\
\psi_{0}(a+b)-\psi_{0}(b)+\mathrm{E}_{p}\left[\log \left(\bar{\gamma}_{2}\right)\right]=0
\end{array}\right.
$$

where $\bar{\gamma}_{2}=\frac{\gamma_{2}}{\gamma_{1}+\gamma_{2}}=1-\bar{\gamma}_{1}$.

Proof: Given in Appendix F.

Remark: The system of equations (16) correspond to equating the expected values of $\log \left(\bar{\gamma}_{1}\right)$ and $\log \left(1-\bar{\gamma}_{1}\right)=\log \left(\bar{\gamma}_{2}\right)$ under both distributions,

$$
\begin{aligned}
\mathrm{E}_{p}\left[\log \left(\bar{\gamma}_{1}\right)\right] & =\mathrm{E}_{q}\left[\log \left(\bar{\gamma}_{1}\right)\right], \\
\mathrm{E}_{p}\left[\log \left(\bar{\gamma}_{2}\right)\right] & =\mathrm{E}_{q}\left[\log \left(\bar{\gamma}_{2}\right)\right] .
\end{aligned}
$$

\section{B. Approximating matrix variate densities}

We present below results on how to approximate matrix variate densities with Wishart and inverse-Wishart densities.

\section{1) Approximation with a $\mathcal{W}$-distribution:}

Theorem 3: Let $p(X)$ be a probability density function defined over $X \in \mathbb{S}_{++}^{d}$. Suppose that $q(X) \triangleq \mathcal{W}_{d}(X ; v, V)$ is the minimizer of $\mathrm{KL}(p \| q)$ among all Wishart densities. Then $V$ is given as

$$
V=\frac{1}{v} \mathrm{E}_{p}[X]
$$

and $v$ is the solution to

$$
\begin{aligned}
& \sum_{i=1}^{d} \psi_{0}((v-i+1) / 2)+d \log (v / 2) \\
& -\mathrm{E}_{p}[\log |X|]+\log \left|\mathrm{E}_{p}[X]\right|=0 .
\end{aligned}
$$

Proof: Given in Appendix G. 
Remark: The expressions for the scale matrix $V$ (18) and degrees of freedom $v$ (19) correspond to equating the expected values of $X$ and $\log |X|$ under both distributions,

$$
\begin{aligned}
\mathrm{E}_{p}[X] & =\mathrm{E}_{q}[X], \\
\mathrm{E}_{p}[\log |X|] & =\mathrm{E}_{q}[\log |X|] .
\end{aligned}
$$

\section{2) Approximation with an $\mathcal{I} \mathcal{W}$-distribution:}

Theorem 4: Let $p(X)$ be a probability density function defined over $X \in \mathbb{S}_{++}^{d}$. Suppose that $q(X) \triangleq \mathcal{I W}_{d}(X ; v, V)$ is the minimizer of $\mathrm{KL}(p \| q)$ among all inverse Wishart distributions. Then $V$ is given as

$$
V=(v-d-1)\left[\mathrm{E}_{p}\left(X^{-1}\right)\right]^{-1}
$$

and $v$ is the solution to

$$
\begin{aligned}
& \sum_{i=1}^{d} \psi_{0}((v-d-i) / 2)-d \log ((v-d-1) / 2) \\
& +\mathrm{E}_{p}(\log |X|)+\log \left|\mathrm{E}_{p}\left(X^{-1}\right)\right|=0 .
\end{aligned}
$$

Proof: Given in Appendix H.

Remark: The expressions for the inverse scale matrix $V$ (21) and degrees of freedom $v$ (22) correspond to equating the expected values of $X^{-1}$ and $\log |X|$ under both distributions,

$$
\begin{aligned}
\mathrm{E}_{p}\left[X^{-1}\right] & =\mathrm{E}_{q}\left[X^{-1}\right], \\
\mathrm{E}_{p}[\log |X|] & =\mathrm{E}_{q}[\log |X|] .
\end{aligned}
$$

\section{Numerical root-finding}

The equations (12), (16), (19), and (22) each have one unique solution, and can be solved using numerical rootfinding, see e.g. [22, Section 5.1]. Examples include NewtonRaphson or modified Newton algorithms, see e.g. [22, Section 5.4], for more alternatives see e.g. [22, Chapter 5].

\section{TARGET COMBINATION}

In this section we address the problem of combination of two extended targets, and describe a methodology that should be applied by a random matrix based Bayesian extended target tracking filter in the case of target combination. In Section IV-A we give a model for extended target combination, and in Section IV-B we show how the combined distribution can be computed, given the combination model and two extended target estimates. In Section IV-C we give a criterion that can be used to determine whether or not two extended target estimates should be combined.

\section{A. Combination model}

The combination of two extended targets $\xi_{k}^{(1)}=$ $\left(\gamma_{k}^{(1)}, \mathbf{x}_{k}^{(1)}, X_{k}^{(1)}\right)$ and $\xi_{k}^{(2)}=\left(\gamma_{k}^{(2)}, \mathbf{x}_{k}^{(2)}, X_{k}^{(2)}\right)$, yielding independent sets of measurements $\mathbf{Z}_{1}$ and $\mathbf{Z}_{2}$, can be seen as the problem of finding the extended target $\xi_{k}=\left(\gamma_{k}, \mathbf{x}_{k}, X_{k}\right)$ that would yield a set of measurements $\mathbf{Z}=\mathbf{Z}_{1} \cup \mathbf{Z}_{2}$, i.e. the union of both measurement sets.
Let $\mathbf{Z}_{1}=\left\{\mathbf{z}_{1}^{(j)}\right\}_{j=1}^{n_{1}}$ and $\mathbf{Z}_{2}=\left\{\mathbf{z}_{2}^{(j)}\right\}_{j=1}^{n_{2}}$ be two sets of measurements, where $\mathbf{z}_{i}^{(j)} \in \mathbb{R}^{d}$ for all $i, j$. The corresponding sample means and sample covariances are given as

$$
\begin{aligned}
\overline{\mathbf{z}}_{i} & =\frac{1}{n_{i}} \sum_{j=1}^{n_{i}} \mathbf{z}_{i}^{(j)}, \\
Z_{i} & =\frac{1}{n_{i}} \sum_{j=1}^{n_{i}}\left(\mathbf{z}_{i}^{(j)}-\overline{\mathbf{z}}_{i}\right)\left(\mathbf{z}_{i}^{(j)}-\overline{\mathbf{z}}_{i}\right)^{\mathrm{T}},
\end{aligned}
$$

for $i=1,2$, respectively. Straightforward calculations will give the following sample mean and sample covariance for $\mathbf{Z}$,

$$
\begin{aligned}
\overline{\mathbf{z}}= & \frac{n_{1}}{n_{1}+n_{2}} \overline{\mathbf{z}}_{1}+\frac{n_{2}}{n_{1}+n_{2}} \overline{\mathbf{z}}_{2}, \\
Z= & \frac{n_{1}}{n_{1}+n_{2}} Z_{1}+\frac{n_{2}}{n_{1}+n_{2}} Z_{2} \\
& +\frac{n_{1} n_{2}}{\left(n_{1}+n_{2}\right)^{2}}\left(\overline{\mathbf{z}}_{1}-\overline{\mathbf{z}}_{2}\right)\left(\overline{\mathbf{z}}_{1}-\overline{\mathbf{z}}_{2}\right)^{\mathrm{T}} .
\end{aligned}
$$

Considering that, under the measurement model (7), $\overline{\mathbf{z}}_{i}$ and $Z_{i}$ are the maximum likelihood estimates of $H \mathbf{x}^{(i)}$ and $X^{(i)}$, an intuitive two target combination model for the kinematical and extension states can be based on (25) as follows,

$$
\begin{aligned}
\mathbf{x}_{k}= & \frac{\gamma_{k}^{(1)}}{\gamma_{k}^{(1)}+\gamma_{k}^{(2)}} \mathbf{x}_{k}^{(1)}+\frac{\gamma_{k}^{(2)}}{\gamma_{k}^{(1)}+\gamma_{k}^{(2)}} \mathbf{x}_{k}^{(2)} \\
X_{k}= & \frac{\gamma_{k}^{(1)}}{\gamma_{k}^{(1)}+\gamma_{k}^{(2)}} X_{k}^{(1)}+\frac{\gamma_{k}^{(2)}}{\gamma_{k}^{(1)}+\gamma_{k}^{(2)}} X_{k}^{(2)} \\
& +\frac{\gamma_{k}^{(1)} \gamma_{k}^{(2)}}{\left(\gamma_{k}^{(1)}+\gamma_{k}^{(2)}\right)^{2}} H\left(\mathbf{x}_{k}^{(1)}-\mathbf{x}_{k}^{(2)}\right)\left(\mathbf{x}_{k}^{(1)}-\mathbf{x}_{k}^{(2)}\right)^{\mathrm{T}} H^{\mathrm{T}} .
\end{aligned}
$$

For the Poisson rate, the sum of two Poisson distributed variables with rates $\gamma_{k}^{(1)}$ and $\gamma_{k}^{(2)}$ is Poisson distributed with rate $\gamma_{k}^{(1)}+\gamma_{k}^{(2)}$. Thus, for the Poisson rate we have the following model,

$$
\gamma_{k}=\gamma_{k}^{(1)}+\gamma_{k}^{(2)}
$$

\section{B. Combined distribution for two extended targets}

Let the states $\xi_{k}^{(1)}$ and $\xi_{k}^{(2)}$ of the two extended targets to be combined be distributed as follows,

$$
\begin{aligned}
& p\left(\xi_{k}^{(1)} \mid \mathbf{Z}^{k}\right)=\mathcal{G} \mathcal{G} \mathcal{I} \mathcal{W}\left(\xi_{k}^{(1)} ; \zeta_{k \mid k}^{(1)}\right), \\
& p\left(\xi_{k}^{(2)} \mid \mathbf{Z}^{k}\right)=\mathcal{G} \mathcal{G} \mathcal{I} \mathcal{W}\left(\xi_{k}^{(2)} ; \zeta_{k \mid k}^{(2)}\right) .
\end{aligned}
$$

We wish to find the parameter $\zeta_{k \mid k}$ of the distribution

$$
p\left(\xi_{k} \mid \mathbf{Z}^{k}\right)=\mathcal{G} \mathcal{G} \mathcal{I} \mathcal{W}\left(\gamma_{k} ; \zeta_{k \mid k}\right),
$$

where $\xi_{k}=\left(\gamma_{k}, \mathbf{x}_{k}, X_{k}\right)$ is the state of the combined extended target and is given by the model (26). In what follows, we use the quantities $\bar{\gamma}_{k}^{1}$ and $\bar{\gamma}_{k}^{2}$ given as

$$
\begin{aligned}
& \bar{\gamma}_{k}^{(1)}=\frac{\gamma_{k}^{(1)}}{\gamma_{k}^{(1)}+\gamma_{k}^{(2)}}, \\
& \bar{\gamma}_{k}^{(2)}=\frac{\gamma_{k}^{(2)}}{\gamma_{k}^{(1)}+\gamma_{k}^{(2)}}=1-\bar{\gamma}_{k}^{(1)},
\end{aligned}
$$


which are distributed with beta distributions obtained in Theorem 2.

1) Poisson rate: A gamma distribution for $\gamma_{k}=\gamma_{k}^{(1)}+\gamma_{k}^{(2)}$ is obtained using Theorem 1.

2) Marginal distribution of kinematical state: Let $\mathbf{m}_{k \mid k}^{(i)}$ and $\hat{P}_{k \mid k}^{(i)}$ be the mean vector and covariance matrix of the Gaussian marginal distribution of $\mathbf{x}_{k}^{(i)}, i=1,2$, see Appendix C. Straightforward calculations show that $p\left(\mathbf{x}_{k} \mid \mathbf{Z}^{k}\right)=\mathcal{N}\left(\mathbf{x}_{k} ; \mathbf{m}_{k \mid k}, \hat{P}_{k \mid k}\right)$, where $\mathbf{m}_{k \mid k}$ and $\hat{P}_{k \mid k}$ are given as

$$
\begin{aligned}
\mathbf{m}_{k \mid k} & =\mathrm{E}\left[\bar{\gamma}_{k}^{(1)}\right] \mathbf{m}_{k \mid k}^{(1)}+\mathrm{E}\left[\bar{\gamma}_{k}^{(2)}\right] \mathbf{m}_{k \mid k}^{(2)} \\
\hat{P}_{k \mid k} & =\mathrm{E}\left[\left(\bar{\gamma}_{k}^{(1)}\right)^{2}\right] \hat{P}_{k \mid k}^{(1)}+\mathrm{E}\left[\left(\bar{\gamma}_{k}^{(2)}\right)^{2}\right] \hat{P}_{k \mid k}^{(2)}
\end{aligned}
$$

The expected values are given in Appendix A.

3) Extension state: Rewrite (26b) as

$$
X_{k}=\bar{\gamma}_{k}^{(1)} X_{k}^{(1)}+\bar{\gamma}_{k}^{(2)} X_{k}^{(2)}+\bar{\gamma}_{k}^{(1)} \bar{\gamma}_{k}^{(2)} X_{k}^{(12)},
$$

where

$$
X_{k}^{(12)}=H\left(\mathbf{x}_{k}^{(1)}-\mathbf{x}_{k}^{(2)}\right)\left(\mathbf{x}_{k}^{(1)}-\mathbf{x}_{k}^{(1)}\right)^{\mathrm{T}} H^{\mathrm{T}},
$$

with expected value and covariance given in Appendix B for the marginal distributions of $\mathbf{x}_{k}^{(i)}$.

Below we are going to find an approximate inverse Wishart density for $X_{k}$ as follows:

1) Approximate the true density of $X_{k}$ with a Wishart distribution. This requires the expected values of $X_{k}$ and $\log \left|X_{k}\right|$. The latter expected value does not have an analytical solution, and must be approximated.

2) Approximate the Wishart distribution with an inverse Wishart distribution. This requires the expected values of $X_{k}^{-1}$ and $\log \left|X_{k}\right|$, which both have analytical solutions under the Wishart distribution obtained in step 1.

The reason that we do not approximate the true density of $X_{k}$ with an inverse Wishart density directly is that this would require us to approximate also the expected value of $X_{k}^{-1}$. With the two step approach outlined above, only one expected value approximation is needed, which, we have empirically found, gives better results.

Using Theorem 3, the distribution over $X_{k}$ can be approximated with a Wishart distribution

$$
p\left(X_{k} \mid \mathbf{Z}^{k}\right) \approx \mathcal{W}_{d}\left(X_{k} ; w_{k \mid k}, W_{k \mid k}\right) .
$$

Theorem 3 requires the expected value of $\log \left|X_{k}\right|$, which does not have an analytical solution. It is approximated using a second order Taylor expansion around $\mathrm{E}\left[X_{k}\right]$. The required first and second order moments of $X_{k}$ are

$$
\begin{aligned}
\mathrm{E}\left[X_{k}\right]= & \mathrm{E}\left[\bar{\gamma}_{k}^{(1)}\right] \mathrm{E}\left[X_{k}^{(1)}\right]+\mathrm{E}\left[\bar{\gamma}_{k}^{(2)}\right] \mathrm{E}\left[X_{k}^{(2)}\right] \\
& +\mathrm{E}\left[\bar{\gamma}_{k}^{(1)} \bar{\gamma}_{k}^{(2)}\right] \mathrm{E}\left[X_{k}^{(12)}\right] \\
\mathrm{E}\left[X_{k, i j} X_{k, m n}\right]= & \mathrm{E}\left[\bar{\gamma}_{1}^{2}\right] \operatorname{Cov}\left(X_{k}^{(1)}\right)_{i j m n} \\
& +\mathrm{E}\left[\bar{\gamma}_{2}^{2}\right] \operatorname{Cov}\left(X_{k}^{(2)}\right)_{i j m n} \\
& +\mathrm{E}\left[\bar{\gamma}_{k}^{(1)} \bar{\gamma}_{k}^{(2)}\right] \operatorname{Cov}\left(X_{k}^{(12)}\right)_{i j m n} \\
& +\mathrm{E}\left[X_{k, i j}\right] \mathrm{E}\left[X_{k, m n}\right]
\end{aligned}
$$

where $X_{k, i j}$ denotes the $i, j$ th element of $X_{k}$ (ith row and $j$ th column), and $\operatorname{Cov}\left(X_{k}\right)_{i j m n}$ denotes the covariance between $X_{k, i j}$ and $X_{k, m n}$. Using Theorem 4, the Wishart distribution (33) is approximated with an inverse Wishart distribution,

$$
p\left(X_{k} \mid \mathbf{Z}^{k}\right) \approx \mathcal{I}_{\mathcal{W}_{d}}\left(X_{k} ; v_{k \mid k}, V_{k \mid k}\right) .
$$

The required expected values of $X_{k}^{-1}$ and $\log \left|X_{k}\right|$, under the Wishart distribution (33), are given in Appendix D.

4) Conditional distribution of kinematical state: The conditional distribution for $\mathbf{x}_{k}$ is

$$
p\left(\mathbf{x}_{k} \mid X_{k}, \mathbf{Z}^{k}\right)=\mathcal{N}\left(\mathbf{x}_{k} ; \mathbf{m}_{k \mid k}, P_{k \mid k} \otimes X_{k}\right),
$$

where $\mathbf{m}_{k \mid k}$ is given in (30). Given $\hat{P}_{k \mid k}$ in (30), and $v_{k \mid k}$ and $V_{k \mid k}$ in (36), $P_{k \mid k}$ is obtained as the least squares solution to

$$
\hat{P}_{k \mid k}=\frac{P_{k \mid k} \otimes V_{k \mid k}}{v_{k \mid k}+s-s d-2} .
$$

Due to the symmetry of all three matrices, this least squares problem has $s(s+1) / 2$ unknown variables and $n_{x}\left(n_{x}+1\right) / 2=$ $s d(s d+1) / 2$ equations, thus the problem is overdetermined.

\section{Target combination criterion}

Two extended targets should be combined into one larger target if (and only if) they are located close to each other, and have similar velocity vectors. We decompose this requirement into two separate criteria, one for the spatial closeness, and one for the velocity vectors.

1) Spatial closeness: Spatial closeness is defined as whether or not the two targets' extensions overlap. Let $\hat{\mathbf{x}}_{k \mid k}^{(i)}=$ $\mathrm{E}\left[\mathbf{x}_{k}^{(i)} \mid \mathbf{Z}^{k}\right]$ and $\hat{X}_{k \mid k}^{(i)}=\mathrm{E}\left[X_{k}^{(i)} \mid \mathbf{Z}^{k}\right]$. A point $\mathbf{p} \in \mathbb{R}^{n_{x}}$ lies within $v>0$ standard deviations of $\hat{\mathbf{x}}_{k \mid k}^{(i)}$ if the following holds,

$$
\left(\mathbf{p}-H \hat{\mathbf{x}}_{k \mid k}^{(i)}\right)^{\mathrm{T}}\left(v^{2} \hat{X}_{k \mid k}^{(i)}\right)^{-1}\left(\mathbf{p}-H \hat{\mathbf{x}}_{k \mid k}^{(i)}\right)<1 .
$$

Let $\mathbf{P}_{i}$ be the set of points $\mathbf{p}$ that satisfy (39).

Overlap of the target extensions $X_{k}^{(i)}$ and $X_{k}^{(j)}$ is here simplified to whether or not the intersection $\mathbf{P}_{i j}=\mathbf{P}_{i} \cap \mathbf{P}_{j}$ is non-empty. This corresponds to the non-existence of a hyperplane that separates the two ellipsoids $\left(\hat{\mathbf{x}}_{k \mid k}^{(i)}, v^{2} \hat{X}_{k \mid k}^{(i)}\right)$ and $\left(\hat{\mathbf{x}}_{k \mid k}^{(j)}, v^{2} \hat{X}_{k \mid k}^{(j)}\right)$, which can be posed as a second order cone program (SOCP) feasibility problem, see e.g. [23, Problem 4.25]. An SOCP feasibility problem is a type of convex optimization problem, and it can be readily solved using standard MATLAB interfaces such as YALMIP [24], [25] or CVX [26], [27]. 
2) Velocity vectors: Two extended targets have similar velocity vectors if the following holds,

$$
\underbrace{\left(\mathbf{m}_{k \mid k}^{(i)}-\mathbf{m}_{k \mid k}^{(j)}\right)^{\mathrm{T}} I_{\mathrm{v}}^{\mathrm{T}} \Lambda_{k \mid k}^{(i j)} I_{\mathrm{v}}\left(\mathbf{m}_{k \mid k}^{(i)}-\mathbf{m}_{k \mid k}^{(j)}\right)}_{c_{i j}^{\mathrm{v}}}<u_{\mathrm{v}},
$$

where $u_{\mathrm{v}}>0$ is a threshold, $\Lambda_{k \mid k}^{(i j)}=\left(\hat{P}_{k \mid k}^{(i)}\right)^{-1}+\left(\hat{P}_{k \mid k}^{(j)}\right)^{-1}$ and $I_{\vee}$ is an $n_{x} \times n_{x}$ matrix with identities on the velocity states (all other elements are zero). This is a modified version of a criterion that was used to group single measurement targets [28].

3) Combination criterion: In order to not combine targets that are close but moving in different directions, or combine targets moving at similar velocity in different parts of the surveillance space, two extended targets are combined if (and only if) the following holds,

$$
\underbrace{\left(\mathbf{P}_{i} \cap \mathbf{P}_{j} \neq \emptyset\right) \&\left(c_{i j}^{\mathrm{v}}<u_{\mathrm{v}}\right)}_{\triangleq_{\operatorname{comb}}\left(\hat{\xi}_{k \mid k}^{(i)}, \hat{\xi}_{k \mid k}^{(j)}\right)}
$$

where $\&$ is the logical and operator.

\section{TARGET SPAWNING}

This section addresses the problem of extended target spawning and describes a methodology that should be applied by a random matrix based Bayesian extended target tracking filter in the case of target spawning. Here we will only consider two target spawning, and we will assume that the spawning event occurs in between measurement generation, i.e. during the prediction step of extended target tracking filtering.

Since there might be many different spawning pairs which, when combined, would give the same original extended target, we will adopt a multiple hypotheses framework where each hypothesis represents an alternative spawning event.

\section{A. Spawning model}

Let the target distribution be

$$
p\left(\xi_{k-1} \mid \mathbf{Z}^{k-1}\right)=\mathcal{G} \mathcal{G} \mathcal{I} \mathcal{W}\left(\xi_{k-1} ; \zeta_{k-1 \mid k-1}\right) .
$$

By means of a prediction update, see [12], [15], [29], a predicted target distribution

$$
p\left(\xi_{k} \mid \mathbf{Z}^{k-1}\right)=\mathcal{G} \mathcal{G} \mathcal{I} \mathcal{W}\left(\xi_{k} ; \zeta_{k \mid k-1}\right),
$$

can be obtained. In case a spawning event takes place during the prediction phase, we would instead have two targets

$$
\begin{aligned}
& p\left(\xi_{k}^{(1)} \mid \mathbf{Z}^{k-1}\right)=\mathcal{G} \mathcal{G I} \mathcal{W}\left(\xi_{k}^{(1)} ; \zeta_{k \mid k-1}^{(1)}\right), \\
& p\left(\xi_{k}^{(2)} \mid \mathbf{Z}^{k-1}\right)=\mathcal{G} \mathcal{G I} \mathcal{W}\left(\xi_{k}^{(2)} ; \zeta_{k \mid k-1}^{(2)}\right) .
\end{aligned}
$$

Assume that the Poisson rates relate to each other as follows,

$$
\begin{aligned}
\gamma_{k}^{(1)} & =\kappa \gamma_{k}, \\
\gamma_{k}^{(2)} & =(1-\kappa) \gamma_{k},
\end{aligned}
$$

where $0<\kappa<1$. Further, assume that the two spawned targets' extensions relate to each other as follows,

$$
\begin{aligned}
& X_{k}^{(1)}=\kappa X_{k}^{(1 / 2)}, \\
& X_{k}^{(2)}=(1-\kappa) X_{k}^{(1 / 2)},
\end{aligned}
$$

i.e. the extensions have the same shape but different size. The matrix $X_{k}^{(1 / 2)} \in \mathbb{S}_{++}^{d}$ is introduced to simplify the notation below. Note that (45) and (46) can be interpreted as meaning that a larger target (i.e. larger extension) will cause more measurements (i.e. have a higher Poisson rate).

If the two spawned targets (44) were to immediately combine into one target, the resulting combined target is assumed to be equal to the prediction (43). Under this assumption, inserting (45) and (46) into the target combination model (26) gives

$$
\begin{aligned}
\mathbf{x}_{k} & =\kappa \mathbf{x}_{k}^{(1)}+(1-\kappa) \mathbf{x}_{k}^{(2)}, \\
X_{k} & =(1+2 \kappa(\kappa-1)) X_{k}^{(1 / 2)}+\kappa(1-\kappa) X_{k}^{(12)}, \\
\gamma_{k} & =\gamma_{k}^{(1)}+\gamma_{k}^{(2)},
\end{aligned}
$$

where $X_{k}^{(12)}$ is defined as in (32). For a given $\kappa,(47)$ is the suggested spawning model.

The assumption that both spawned targets have the same shape, cf. (46), is limiting, however it is necessary because we have two unknown variables, $X_{k}^{(1)}$ and $X_{k}^{(2)}$, and only one equation (26b). Furthermore, the assumption is not very critical because it is made in the prediction step, and the subsequent correction step(s) would correct the shapes.

\section{B. Spawning hypotheses}

Given a prior target distribution (42), the prediction method from [12], [16] is used to obtain the predicted target distribution (43). Note that, for a given predicted target distribution (43), there exists an infinite number of spawning pairs (44) whose combination is identical to the predicted single target.

We generate multiple spawning hypotheses as follows. For each $\kappa$ value, and each dimension $\ell$ of the extension, one spawned estimate pair is generated, with parameters $\zeta_{k \mid k-1}^{(1, \ell, \kappa)}$ and $\zeta_{k \mid k-1}^{(2, \ell, \kappa)}$.

1) Poisson rates: It follows from the definition of $\mathcal{G} \mathcal{A M}(\cdot)$ that $\gamma_{k}^{(1)}$ and $\gamma_{k}^{(2)}$ are gamma distributed with parameters

$$
\begin{aligned}
& \alpha_{k \mid k-1}^{(i, \ell, \kappa)}=\alpha_{k \mid k-1} \text { for } i=1,2, \\
& \beta_{k \mid k-1}^{(1, \ell, \kappa)}=\frac{\beta_{k \mid k-1}}{\kappa} \\
& \beta_{k \mid k-1}^{(2, \ell, \kappa)}=\frac{\beta_{k \mid k-1}}{1-\kappa} .
\end{aligned}
$$

2) Kinematical states: Let $\hat{X}_{k \mid k-1}=\mathrm{E}\left[X_{k} \mid \mathbf{Z}^{k-1}\right]$ under the pdf (43), and let $e_{\ell}$ and $\mathbf{v}_{\ell}$ be the $\ell$ :th eigenvalue and eigenvector of $\hat{X}_{k \mid k-1}$. We set the parameters of the spawned kinematical states to

$$
\begin{aligned}
\mathbf{m}_{k \mid k-1}^{(1, \ell, \kappa)} & =\mathbf{m}_{k \mid k-1}+(1-\kappa) \sqrt{e_{\ell}} H^{\mathrm{T}} \mathbf{v}_{\ell}, \\
\mathbf{m}_{k \mid k-1}^{(2, \ell, \kappa)} & =\mathbf{m}_{k \mid k-1}-\kappa \sqrt{e_{\ell}} H^{\mathrm{T}} \mathbf{v}_{\ell}, \\
P_{k \mid k-1}^{(i, \ell, \kappa)} & =P_{k \mid k-1} \text { for } i=1,2 .
\end{aligned}
$$


Note that other ways are possible, however, empirically we have found that (49) gives good results.

3) Extension states: Rewriting (47b), we have

$$
X_{k}^{(1 / 2)}=\underbrace{\frac{1}{1+2 \kappa(\kappa-1)}}_{\triangleq \kappa_{1}} X_{k}-\underbrace{\frac{\kappa(1-\kappa)}{1+2 \kappa(\kappa-1)}}_{\triangleq \kappa_{2}} X_{k}^{(12)} \text {. }
$$

Similarly to Section IV-B3, we first approximate the true distribution over $X_{k}^{(1 / 2)}$ with a Wishart distribution, and subsequently approximate the Wishart distribution with an inverse Wishart distribution.

Using Theorem 3 the distribution over $X_{k}^{(1 / 2)}$ is approximated with a Wishart distribution

$$
p\left(X_{k}^{(1 / 2)} \mid \mathbf{Z}^{k-1}\right) \approx \mathcal{W}_{d}\left(X_{k}^{(1 / 2)} ; w_{k \mid k-1}^{(\ell, \kappa)}, W_{k \mid k-1}^{(\ell, \kappa)}\right) .
$$

This requires the expected value of $\log \left|X_{k}^{(1 / 2)}\right|$, for which there is no analytical solution. As in Section IV-B3, the expected value is approximated using a second order Taylor expansion around $\mathrm{E}\left[X_{k}^{(1 / 2)}\right]$. The necessary first and second order moments of $X_{k}^{(1 / 2)}$ are

$$
\begin{aligned}
\mathrm{E}\left[X_{k}^{1 / 2}\right]= & \kappa_{1} \mathrm{E}\left[X_{k}\right]-\kappa_{2} \mathrm{E}\left[X_{k}^{(12)}\right], \\
\mathrm{E}\left[X_{k, i j}^{(1 / 2)} X_{k, m n}^{(1 / 2)}\right]= & \kappa_{1}^{2} \operatorname{Cov}\left(X_{k}\right)_{i j m n}+\kappa_{2}^{2} \operatorname{Cov}\left(X_{k}^{(12)}\right)_{i j m n} \\
& +\mathrm{E}\left[X_{k, i j}^{(1 / 2)}\right] \mathrm{E}\left[X_{k, m n}^{(1 / 2)}\right] .
\end{aligned}
$$

The distribution (51) is subsequently approximated with an inverse Wishart distribution using Theorem 4,

$$
p\left(X_{k}^{(1 / 2)} \mid \mathbf{Z}^{k-1}\right) \approx \mathcal{I W}_{d}\left(X_{k}^{(1 / 2)} ; v_{k \mid k-1}^{(\ell, \kappa)}, V_{k \mid k-1}^{(\ell, \kappa)}\right) .
$$

Finally, by [14, Theorems 3.3.11 and 3.4.1] we have

$$
\begin{aligned}
& p\left(X_{k}^{(1)} \mid \mathbf{Z}^{k-1}\right) \approx \mathcal{I W}_{d}\left(X_{k}^{(1)} ; v_{k \mid k-1}^{(\ell, \kappa)}, \kappa V_{k \mid k-1}^{(\ell, \kappa)}\right), \\
& p\left(X_{k}^{(2)} \mid \mathbf{Z}^{k-1}\right) \approx \mathcal{I}_{d}\left(X_{k}^{(2)} ; v_{k \mid k-1}^{(\ell, \kappa)},(1-\kappa) V_{k \mid k-1}^{(\ell, \kappa)}\right) .
\end{aligned}
$$

4) Summary: To summarize, for each dimension $\ell$ of the extension and each $\kappa$ value, a spawned estimate pair is generated with the following parameters

$\zeta_{k \mid k-1}^{(1, \ell, \kappa)}=\left(\alpha_{k \mid k-1}, \frac{\beta_{k \mid k-1}}{\kappa}, \mathbf{m}_{k \mid k-1}^{(1, \ell, \kappa)}, P_{k \mid k-1}^{(1, \ell, \kappa)}, v_{k \mid k-1}^{(\ell, \kappa)}, \kappa V_{k \mid k-1}^{(\ell, \kappa)}\right)$,

$\zeta_{k \mid k-1}^{(2, \ell, \kappa)}=\left(\alpha_{k \mid k-1}, \frac{\beta_{k \mid k-1}}{1-\kappa}, \mathbf{m}_{k \mid k-1}^{(2, \ell, \kappa)}, P_{k \mid k-1}^{(2, \ell, \kappa)}, v_{k \mid k-1}^{(\ell, \kappa)},(1-\kappa) V_{k \mid k-1}^{(\ell, \kappa)}\right)$

If a set $\mathbf{K}$ of $K$ different $\kappa$ values are used, in total $d K$ spawned estimate pairs are generated, or $2 d K$ GGIW components.

\section{ON THE USE OF OTHER SPATIAL DISTRIBUTIONS}

By using positive definite matrices to represent the target extensions our work implicitly assumes that the target extent is an ellipsoid. Moreover, the spatial distribution of the measurements in our work is a Gaussian density. One potential extension of the presented work is thus to relax the Gaussian and/or the ellipsoidal assumption. This would allow different types of spatial distributions for the target measurements, see e.g. [30].

The methodology presented here gives hints on what type of approach can be used in a general setting, e.g. when parametric densities from the exponential family are used. The proposed combination model is based on representing the set of measurements, generated by the individual target's spatial densities, with a single spatial density of the same functional form as those of the individual targets. With a different parametric spatial density, one would need to write the formulae for the combined density parameters in terms of the formulae that connect the parameters of the spatial density of each target to the corresponding measurements. This is what is performed in (24) and (25).

The multiple hypothesis methodology for spawning could also be useful if other spatial distributions are used. In this work a single ellipsoid is simply divided into alternative possible ellipsoids, if other distributions from the exponential family are used, similar division methods must be devised. If the spatial distribution is multi-modal, the different modes of the spatial density might provide intuitive alternative divisions. Note that, in the spawning case and without using the subsequent measurements, one can never arrive at a unique solution for how a single target can be divided into multiple targets. Therefore, an uncertainty margin must always be left for the forthcoming measurements to resolve.

\section{MULTIPLE TARGET TRACKING FRAMEWORK}

To demonstrate the merits of the presented methodologies for target combination and target spawning, the methodologies must be integrated into a multiple extended target tracking framework. In this section we will briefly describe the framework that we have worked in, we show how combination and spawning fits into the framework, and we also discuss target extraction and performance metrics.

\section{A. The GGIW-PHD filter}

We have used a modified version of the Gaussian inverse Wishart (GIW) implementation [29], [31] of the extended target probability hypothesis density (PHD) filter proposed by Mahler [32]. In the GIW-PHD filter the extended target state is composed only of the kinematical and extension states (i.e. there are no Poisson rates), and the PHD of the target set is approximated as a mixture of GIW densities as follows [29]

$$
\begin{aligned}
D_{k \mid k}\left(\xi_{k}\right) \approx \sum_{j=1}^{J_{k \mid k}} w_{k \mid k}^{(j)} & \mathcal{N}\left(\mathbf{x}_{k} ; \mathbf{m}_{k \mid k}^{(j)}, P_{k \mid k}^{(j)} \otimes X_{k}\right) \\
& \times \mathcal{I} \mathcal{W}_{d}\left(X_{k} ; \nu_{k \mid k}^{(j)}, V_{k \mid k}^{(j)}\right),
\end{aligned}
$$

where $J_{k \mid k}$ is the number of mixture components, and the scalars $w_{k \mid k}^{(j)}>0$ are the components weights.

In the modified version of the GIW-PHD filter that we use in the current work, called the GGIW-PHD filter, the extended target state also includes the Poisson rates. The PHD of the 
TABLE I

GGIW-PHD FILTER TARGET COMBINATION

1: require: Combination criterion parameters $v$ and $u_{\mathrm{v}}$, and PHD intensity

$$
D_{k \mid k}\left(\xi_{k}\right)=\sum_{j=1}^{J_{k \mid k}} w_{k \mid k}^{(j)} \mathcal{G} \mathcal{G} \mathcal{I} \mathcal{W}\left(\xi_{k} ; \zeta_{k \mid k}^{(j)}\right) \text {. }
$$

2: initialize: $\mathbf{I}=\left\{i \mid w_{k \mid k}^{(i)} \geq 0.5\right\}$,

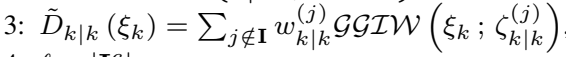

4: $\ell=\left|\mathbf{I}^{c}\right|$.

5: repeat

6: $\quad \ell=\ell+1$

7: $\quad j=\underset{i \in \mathbf{I}}{\arg \max } w_{k \mid k}^{(i)}$

8: $\quad \mathbf{I}_{j}=\left\{i \in \mathbf{I} \backslash j \mid \operatorname{comb}\left(\hat{\xi}_{k \mid k}^{(i)}, \hat{\xi}_{k \mid k}^{(j)}\right)=1\right\}$

if $\mathbf{I}_{j} \neq \emptyset$ then

$n=\underset{i \in \mathbf{I}_{j}}{\arg \max } w_{k \mid k}^{(i)}$

10

Combine components $j$ and $n$ as presented in Section IV-B, let $\tilde{\zeta}_{k \mid k}^{(\ell)}$ denote the corresponding GGIW distribution parameters.

12:

$\tilde{w}_{k \mid k}^{(\ell)}=\mathrm{E}\left[\bar{\gamma}_{k}^{(j)}\right] w_{k \mid k}^{(j)}+\mathrm{E}\left[\bar{\gamma}_{k}^{(n)}\right] w_{k \mid k}^{(j)}$

$$
\mathbf{I}=\mathbf{I} \backslash\{j, n\}
$$

else

$\tilde{\zeta}_{k \mid k}^{(\ell)}=\zeta_{k \mid k}^{(j)}$

$\tilde{w}_{k \mid k}^{(\ell)}=w_{k \mid k}^{(j)}$

$\mathbf{I}=\mathbf{I} \backslash j$

end if

until $\mathbf{I}=\emptyset$

output: Combined PHD intensity, where $\tilde{J}_{k \mid k} \leq J_{k \mid k}$,

$$
\tilde{D}_{k \mid k}\left(\xi_{k}\right)=\sum_{j=1}^{\tilde{J}_{k \mid k}} \tilde{w}_{k \mid k}^{(j)} \mathcal{G} \mathcal{G} \mathcal{I} \mathcal{W}\left(\xi_{k} ; \tilde{\zeta}_{k \mid k}^{(j)}\right) .
$$

target set is approximated as a mixture of GGIW densities as follows

$$
\begin{aligned}
& D_{k \mid k}\left(\xi_{k}\right) \approx \sum_{j=1}^{J_{k \mid k}} w_{k \mid k}^{(j)} \mathcal{G} \mathcal{A} \mathcal{M}\left(\gamma_{k} ; \alpha_{k \mid k}, \beta_{k \mid k}\right) \\
& \times \mathcal{N}\left(\mathbf{x}_{k} ; \mathbf{m}_{k \mid k}^{(j)}, P_{k \mid k}^{(j)} \otimes X_{k}\right) \\
& \times \mathcal{I} \mathcal{W}_{d}\left(X_{k} ; \nu_{k \mid k}^{(j)}, V_{k \mid k}^{(j)}\right) \\
&= \sum_{j=1}^{J_{k \mid k}} w_{k \mid k}^{(j)} \\
& \mathcal{G} \mathcal{G} \mathcal{I} \mathcal{W}\left(\xi_{k} ; \zeta_{k \mid k}^{(j)}\right)
\end{aligned}
$$

In both PHD filter implementations, the parameters of the PHDs are predicted and updated recursively with the measurements. For details on the implementations, please refer to [16], [29], [31].

\section{B. Combination in the GGIW-PHD filter}

Target combination in the GGIW-PHD filter is performed after the correction step (measurement update). An algorithm for target combination is given in Table I. In the algorithm, all GGIW components with a weight less than 0.5 are left unaltered. The components with weight larger than 0.5 are checked for combination in a pairwise manner, starting with the highest weights. Note that any component is combined with at most one other component.
TABLE II

GGIW-PHD FILTER PREDICTION WITH SPAWNING

1: require: Spawning weight $w_{\mathrm{sp}}$, set $\mathbf{K}$ of $\kappa$ values, and PHD intensity

$$
D_{k \mid k}\left(\xi_{k}\right)=\sum_{j=1}^{J_{k \mid k}} w_{k \mid k}^{(j)} \mathcal{G} \mathcal{G} \mathcal{I} \mathcal{W}\left(\xi_{k} ; \zeta_{k \mid k}^{(j)}\right) .
$$

$$
\begin{aligned}
& \text { : initialize: } J_{\mathrm{aux}}=J_{k \mid k} \\
& 3 \text { : for } j=1, \ldots, J_{k \mid k} \text { do } \\
& \text { Predict } j \text { :th component as outlined in [29], [31]. } \\
& \text { if } w_{k \mid k}^{(j)}>0.5 \text { then } \\
& \text { for } \kappa \in \mathbf{K} \text { do } \\
& \text { for } \ell=1, \ldots, d \text { do } \\
& \text { Compute } \zeta_{k \mid k-1}^{(1, \ell, \kappa)} \text { and } \zeta_{k \mid k-1}^{(2, \ell, \kappa)} \text { as presented in Section V-B. } \\
& \text { For } i=1,2 \text {, set } \\
& \begin{aligned}
w_{k+1 \mid k}^{\left(J_{\mathrm{aux}}+i\right)} & =w_{\mathrm{sp}} w_{k \mid k}^{(j)} \\
\zeta_{k+1 \mid k}^{\left(J_{\mathrm{aux}}+i\right)} & =\zeta_{k+1 \mid k}^{(i, \ell, \kappa)}
\end{aligned} \\
& \text { 10: } \quad J_{\text {aux }}=J_{\text {aux }}+2 \\
& \text { 11: } \quad \text { end for } \\
& \text { 12: end for } \\
& \text { 13: end if } \\
& \text { 14: end for } \\
& D_{k+1 \mid k}\left(\xi_{k+1}\right)=\sum_{j=1}^{J_{k+1 \mid k}} w_{k+1 \mid k}^{(j)} \mathcal{G} \mathcal{G} \mathcal{I} \mathcal{W}\left(\xi_{k+1} ; \zeta_{k+1 \mid k}^{(j)}\right),
\end{aligned}
$$

\section{Spawning in the GGIW-PHD filter}

Generation of spawning estimate pairs in the GGIW-PHD filter is performed in the prediction step (time update). An algorithm for target prediction with spawning is given in Table II. The spawning weight parameter $w_{\text {sp }}>0$ can be understood as follows. If the PHD has $\hat{N}_{x, k}$ GGIW components, all with weight $\approx 1$, the total sum of weights for the spawning components is approximately

$$
\hat{N}_{x, k} \times 2 d K \times w_{\text {sp }}=N_{\text {sp }}
$$

The quantity $N_{\text {sp }}$ approximates the mean number of spawned targets. Thus, the more likely spawning events are thought to be, the larger the spawning weight parameter should be set.

In the algorithm, for each component with weight greater than $0.5, K$ additional component pairs (which have negligible weights compared to the corresponding component, because typically $w_{\text {sp }} \ll 1$ ) are added to the predicted PHD. These added components correspond to a heuristic modification of the extended target PHD filter to include spawning hypotheses. The procedure of adding component pairs is analogous to the Gaussian Mixture PHD-filter for point targets [33], in which a single spawned Gaussian component is added for each existing component.

\section{Performance Evaluation}

Let the true target set at time $t_{k}$ be $\mathbf{X}_{k}=\left\{\xi_{k}^{(i)}\right\}_{i=1}^{N_{x, k}}$, where the true target cardinality $N_{x, k}$, and each true target state $\xi_{k}^{(i)}$, are unknown. Estimates of the target states $\hat{\xi}_{k \mid k}^{(j)}$ are obtained by extracting the GGIW components whose weights are larger 
than or equal to a threshold, e.g. 0.5, see [33]. Let the set of extracted targets be denoted

$$
\begin{aligned}
\hat{\mathbf{X}}_{k \mid k} & =\left\{\hat{\xi}_{k \mid k}^{(i)}\right\}_{i=1}^{\hat{N}_{x, k}}, \hat{\xi}_{k \mid k}^{(i)}=\left(\hat{\gamma}_{k \mid k}^{(i)}, \hat{\mathbf{x}}_{k \mid k}^{(i)}, \hat{X}_{k \mid k}^{(i)}\right), \\
\hat{\gamma}_{k \mid k}^{(i)} & =\mathrm{E}\left[\gamma_{k}\right], \hat{\mathbf{x}}_{k \mid k}^{(i)}=\mathrm{E}\left[\mathbf{x}_{k}\right], \hat{X}_{k \mid k}^{(i)}=\mathrm{E}\left[X_{k}\right],
\end{aligned}
$$

where the expected values are taken with respect to the $i$ :th GGIW distribution.

An assignment $\bar{\pi}$ between the true target states $\xi_{k}^{(j)}$ and the extracted states $\hat{\xi}_{k \mid k}^{(i)}$ is computed using the optimal subpattern assignment (OSPA) metric [34]. The tracking results are evaluated in terms of the following quantities,

$$
\begin{aligned}
d^{(\gamma)} & =\sum_{j}\left|\gamma_{k}^{(j)}-\hat{\gamma}_{k \mid k}^{(\bar{\pi}(j))}\right|, \\
d^{(\mathbf{x})} & =\sum_{j}\left\|\mathbf{x}_{k}^{(j)}-\hat{\mathbf{x}}_{k \mid k}^{(\bar{\pi}(j))}\right\|_{2}, \\
d^{(X)} & =\sum_{j}\left\|X_{k}^{(j)}-\hat{X}_{k \mid k}^{(\bar{\pi}(j))}\right\|_{F},
\end{aligned}
$$

where $|\cdot|$ is the absolute value, $\|\cdot\|_{2}$ is the Euclidean norm, and $\|\cdot\|_{F}$ is the Frobenius norm. An estimate of the target cardinality is given by the sum of weights [3], $\hat{N}_{k \mid k}=\sum_{j=1}^{J_{k \mid k}} w_{k \mid k}^{(j)}$.

\section{Simulation STUDY}

This section presents a simulation study conducted for testing the proposed target combination and spawning functions.

\section{A. Multiple target tracking setup}

Four scenarios were simulated. The kinematical state contains $2 D$ position, velocity and acceleration, the extension is two dimensional (i.e. $d=2, n_{x}=6$ and thus $s=3$ ). In each scenario, the $i$ :th target's true extension is $X_{k}^{(i)}=$ $R_{k}^{(i)} \operatorname{diag}\left(\left[\begin{array}{ll}\bar{a}_{i}^{2} & a_{i}^{2}\end{array}\right]\right)\left(R_{k}^{(i)}\right)^{\mathrm{T}}$, where $\bar{a}_{i}$ and $a_{i}$ are the major and minor axes, and $R_{k}^{(i)}$ is a rotation matrix applied such that either $\bar{a}_{i}$ or $a_{i}$ is aligned with the direction of motion. The motion model used in the filter is described in detail in [12], as in [29] the motion model parameters were set to $t_{s}=1 \mathrm{~s}$, $\theta=1 \mathrm{~s}, \Sigma=0.1 \mathrm{~m} / \mathrm{s}^{2}$ and $\tau=5 \mathrm{~s}$.

The true target motions were not generated using a specific motion model. This choice may seem simplistic, however the main focus of this paper is not on motion modeling, but on spawning and combination. The generated true tracks are sufficiently realistic to test the presented spawning and combination functions.

In each scenario a Poisson distributed number of clutter measurements were distributed uniformly in the surveillance space, with Poisson rate 10 per scan.

\section{B. True target tracks}

1) Target combination: In the first scenario two targets maneuver such that they move in parallel and give rise to unresolved sets of measurements, see the true tracks in Figure 1a. The scenario is meant to simulate a real world scenario such as a radar tracking two airplanes that begin to fly in a close formation. It has 24 time steps, starting at time step 12 the targets move in parallel at equal speeds, with their $2 \sigma$ ellipses touching ${ }^{3}$. True target measurements were generated with $\gamma_{k}^{(i)}=20, \bar{a}_{i}=10$ and $a_{i}=5$ for $i=1,2$.

2) Target split: In the second scenario an extended target splits in half into two smaller extended targets, see the true tracks in Figure 1b. The scenario is meant to simulate a real world scenario such as a radar tracking two airplanes flying in close formation before separating. It has 15 time steps, the spawning occurs between time steps 5 and 6 . True target measurements were generated with $\gamma_{k}=40, \bar{a}=10$ and $a=10$ before spawning, and $\gamma_{k}^{(i)}=20, \bar{a}_{i}=10$ and $a_{i}=5$, for $i=1,2$, after spawning.

3) New target appearance: In the third scenario a new smaller target appears next to an existing target, see the true tracks in Figure 1c. The scenario has 15 time steps, the spawning occurs between time steps 5 and 6 . This scenario is meant to simulate a real world scenario such as a radar tracking an airplane that launches a weapon. True target measurements were generated with $\gamma_{k}^{(1)}=40, \bar{a}_{1}=20$ and $a_{1}=5$ for the larger target, and $\gamma_{k}^{(2)}=10, \bar{a}_{2}=6.67$ and $a_{2}=1$ for the smaller spawned target.

4) Target occlusion: In the fourth scenario two targets of different size move in opposite direction towards each other, and as the targets pass each other the smaller target is occluded by the larger target, i.e. it is not seen by the sensor and thus does not produce any measurements. The scenario has 101 time steps, and the true kinematic positions were generated such that $\mathbf{x}_{51}^{(1)}=\mathbf{x}_{51}^{(2)}$, i.e. the targets are at the same position at the 51:st time step. The respective initial positions vary with the simulated constant speed $\varsigma^{(i)}$ of the targets. In Figure 1d the true tracks are shown for $\varsigma^{(i)}=1$. Only every 25 :th time step is shown for increased clarity.

The spawning event occurs when the smaller target becomes visible to the sensor again. Because this happens gradually, it is not possible to give a definitive time for when the spawning happens. The scenario is meant to simulate a real world scenario such as a camera that is used to track two persons moving across the field of view, in opposite directions, and at different distances from the sensor. For the detections in the image plane, this would appear as two different sized targets that move "through" each other.

True target measurements were generated with $\gamma_{k}^{(1)}=30$, $\bar{a}_{1}=10$ and $a_{1}=5$ for the larger target, and $\gamma_{k}^{(2)}=15$, $\bar{a}_{2}=8$ and $a_{2}=3$ for the smaller target. At each time step measurements were simulated for both targets, however for the second target the measurements that fell inside the $3 \sigma$ ellipse of the first target were removed to simulate the occlusion.

\section{Combination results}

For the spatial closeness criterion we set $v=2$, and for the velocity vectors we set $u_{\mathrm{v}}=50$. The results are shown in Figure 2. When the targets are sufficiently close, moving in the same direction, they are combined into just one target.

\footnotetext{
${ }^{3}$ This corresponds to $v=2$ in (39).
} 


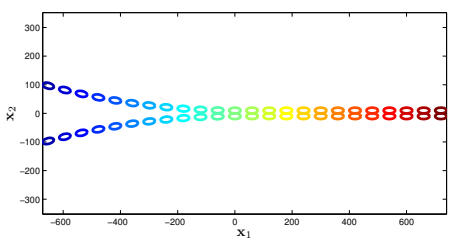

(a)

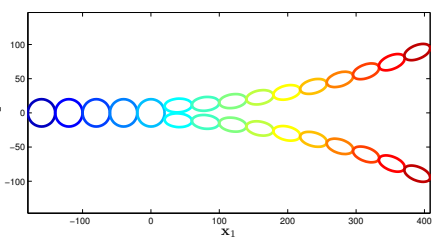

(b)

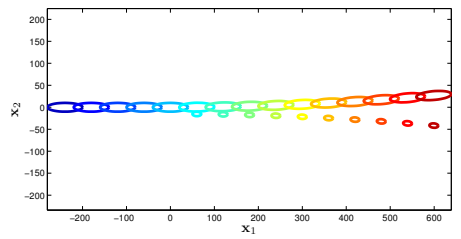

(c)

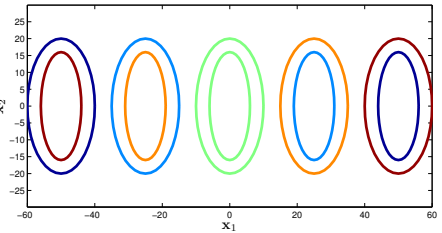

(d)

Fig. 1. True tracks for the simulation scenarios. Colors are used to show time steps, dark blue and dark red are the first and last time steps. (a) (b), (c) and (d) show the true target positions for target combination, target split, new target appearance, and target occlusion, respectively. For the target occlusion scenario, only every 25:th time step is shown for increased clarity.

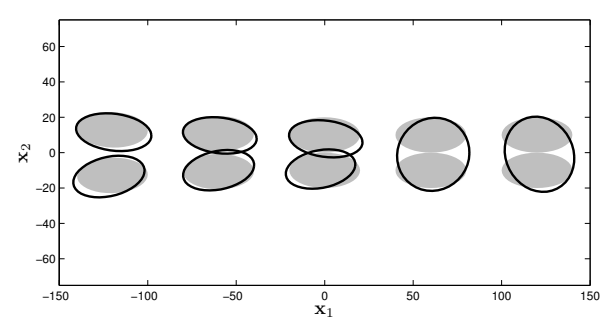

(a)

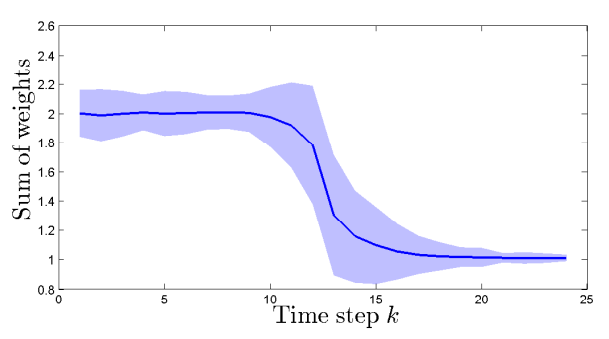

(b)

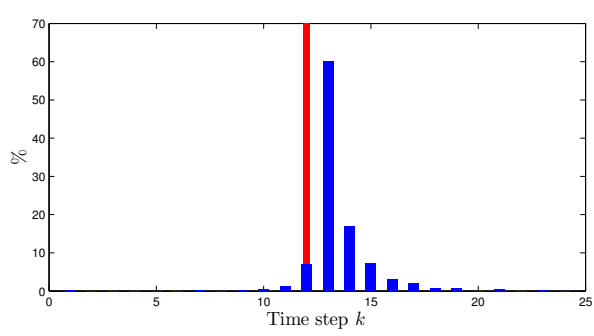

(c)

Fig. 2. Combination of two targets. (a) Example result from a single simulation run. The true targets are shown as the light gray filled ellipses, the target estimates are shown as black ellipses. When the targets are sufficiently close, and have similar velocity vectors, they are combined into one target. (b) Sum of weights (i.e. estimated cardinality), averaged over $10^{3}$ runs, shown in blue. Mean \pm one standard deviation is shown in light blue. (c) Histogram showing for which time step the two targets were combined. The two targets move in parallel starting at time step 12 (red line), in $60 \%$ of the $10^{3}$ simulations the targets estimates were combined after measurement updating in time step 13.

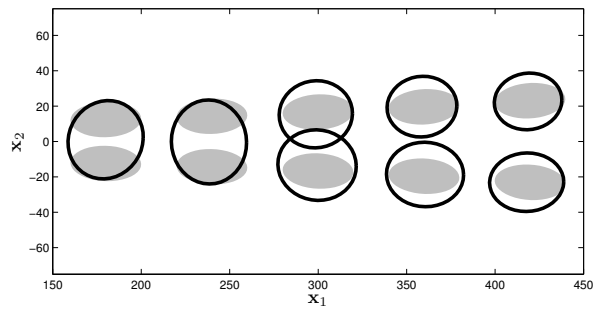

(a)

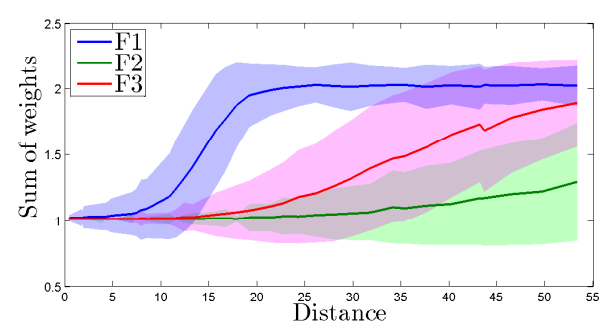

(b)

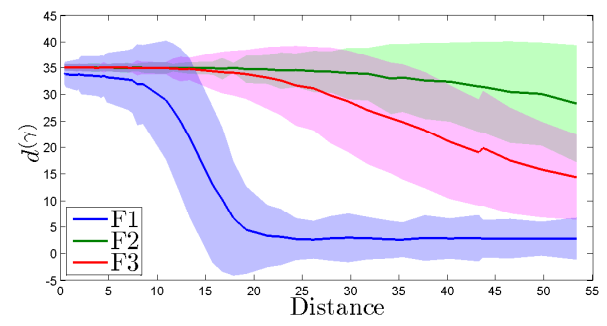

(c)

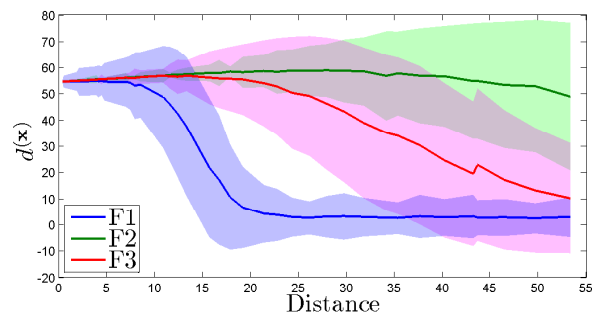

(d)

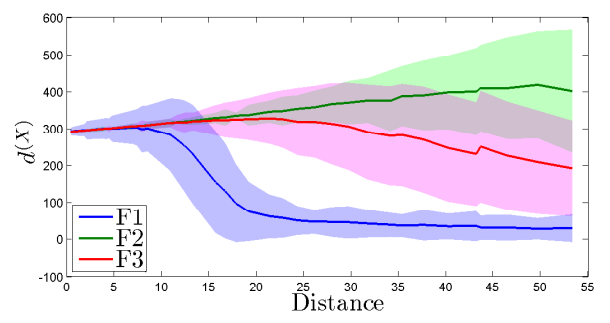

(e)

Fig. 3. Spawning results for the true tracks in Figure 1b. (a) Example result from a single simulation run. The true targets are shown as the light gray filled ellipses, the target estimates are shown as black ellipses. (b) Estimated cardinality (true cardinality is two). (c) Measurement rate estimation error. (d) Kinematic state estimation error. (c) Extension state estimation error. The results in (b) to (e) are averaged over $10^{3}$ Monte Carlo runs, and are shown for different separation distances. While the GGIW-PHD with spawning can detect the spawning events, adding spawned estimate pairs allows the filter to detect the spawning at a closer distance.

For $v=2$, the true targets fulfill the combination criterion between time steps $k=12$ and $k=24$. Over $10^{3}$ Monte Carlo simulations, for $60 \%$ of the cases the two target estimates are combined at time step $k=13$, i.e. with a delay of one time step. The delay is typically caused by the fact that the velocity vector estimates must converge to similar values first.

\section{Spawning results}

Three GGIW-PHD filters were run in parallel: one filter with spawning hypotheses computed using the model presented in Section V (denoted F1), one filter without a spawning model (denoted F2), and one filter with a single spawning hypothesis as in [13] (denoted F3). Neither filter used the 


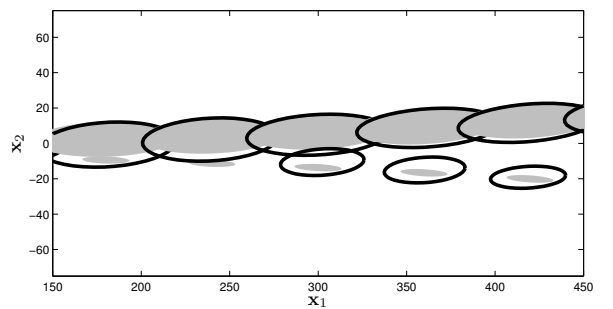

(a)

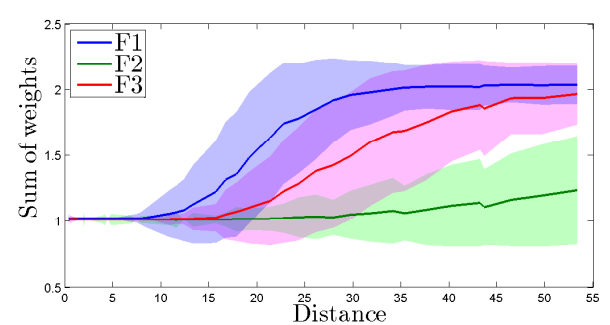

(b)

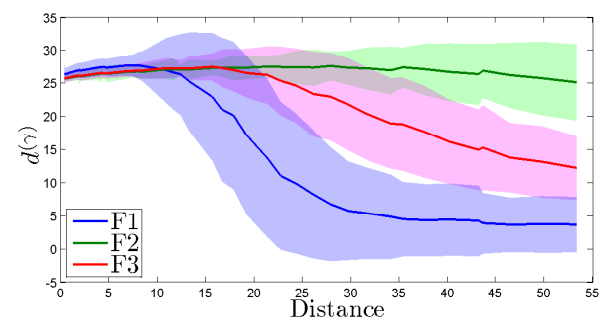

(c)

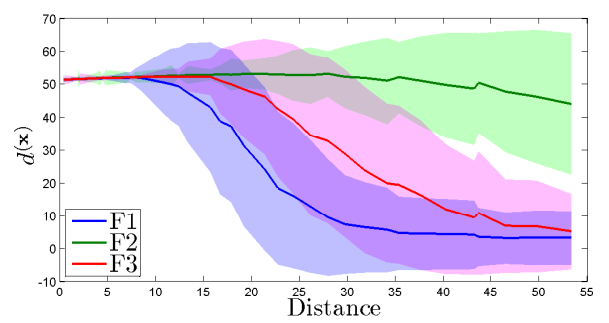

(d)

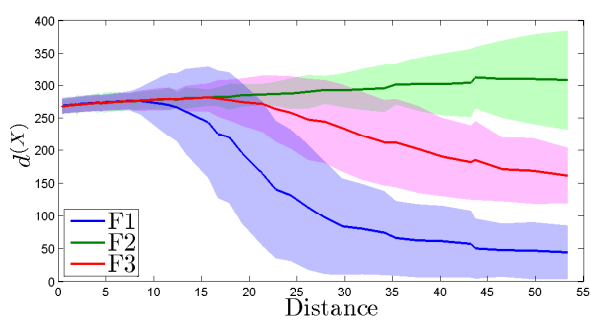

(e)

Fig. 4. Spawning results for the true tracks in Figure 1c. (a) Example result from a single simulation run. The true targets are shown as the light gray filled ellipses, the target estimates are shown as black ellipses. (b) Estimated cardinality (true cardinality is two). (c) Measurement rate estimation error. (d) Kinematic state estimation error. (c) Extension state estimation error. The results in (b) to (e) are averaged over $10^{3}$ Monte Carlo runs, and are shown for different separation distances. While the GGIW-PHD with spawning can detect the spawning events, adding spawned estimate pairs allows the filter to detect the spawning at a closer distance.

target combination outlined in Section IV. In F1 and F3 the spawning weight was $w_{\mathrm{sp}}=0.05$. In F1 spawning hypotheses were generated for

$$
\kappa \in \mathbf{K}=\left\{\frac{1}{4}, \frac{1}{2}, \frac{3}{4}\right\} .
$$

The parameters of F3 were set such that the expected value was constant for the extended target state, and the variance was increased. The variance of the measurement rate was increased by $50 \%$, a matrix $\operatorname{diag}([1,0,0])$ was added to the kinematical state covariance, and the degrees of freedom of the extension state was decreased by 25 . These parameters were chosen such that the best possible performance was obtained.

1) Target split and new target appearance: The second and third scenarios were simulated $10^{3}$ times each, Figures 3 and 4 show the results. The mean sum of weights, and the performance metrics (60), are shown for different distances between the kinematical positions. When the extended targets are still very close, no filter is able to detect the spawning event. However, when the targets start to separate, F1 detects the event at a shorter distance, or equivalently at an earlier time step, than F3. The worst performance is obtained with $\mathrm{F} 2$, i.e. the filter without any spawning model.

There is also a significant difference between the three filters with respect to the performance metrics (60), with F1 clearly having the best performance. After the spawning event is detected by F1 and F3, the measurement rate and kinematical state starts to converge towards the correct value. The extension state has a small positive error, however this is expected. As the two targets turn away from each other, their corresponding extensions rotate, and the simple extension prediction used, see [12], does not account for rotations. As noted in previous work [29], during maneuvers the extension estimation error is always larger than during straight line motion.

2) Target occlusion: The fourth scenario was simulated with different target speeds,

$$
\varsigma^{(i)}=[0.5,0.51, \ldots, 1.0], i=1,2 .
$$

For each speed, the scenario was simulated $10^{2}$ times. The mean estimated cardinalities of all three filters are shown for different target speeds and target distances in Figures 5a, 5b, and $5 \mathrm{c}$, respectively. Figure $5 \mathrm{~d}$ illustrates the contour plots for Figures 5a, 5b, and 5c, superposed onto each other.

As the two targets approach each other, all three filters can track both targets until the point where the targets' respective $1 \sigma$ ellipses are touching. After this point, all three filters estimate cardinality to one target, which is expected. As the targets move away from each other, F1 correctly estimates the cardinality as two around a point which corresponds to when the $4 \sigma$ ellipses of the targets are touching, regardless of the target speed $\varsigma_{k}^{(i)}$. The filter F2 corrects the cardinality estimate at a much later point, especially at lower speeds, and the performance of F3 is inbetween F1 and F2.

This strange dependence of the spawning performance on the target speeds observed in F2, and to a lesser degree also in F3, deserves an explanation. When the second target is occluded, all three filters estimate a single target. Hence when the targets start to separate after the occlusion event, F2 predicts and expects a single target in the next sampling instant. On the other hand, F1 and F3 also expect a single target with large probability, however with small probability, F1 and F3 also expect two targets thanks to the spawning hypotheses their PHDs contains. As the targets separate further, one of the spawning hypotheses gains weight and eventually 


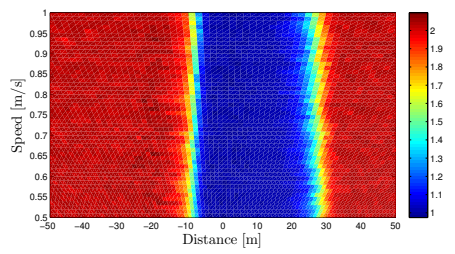

(a)

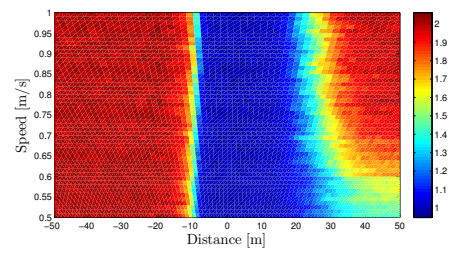

(b)

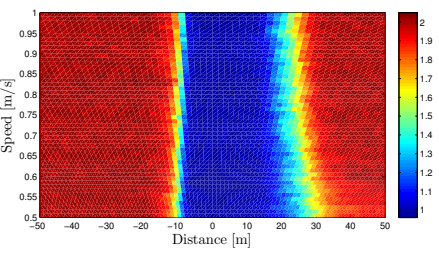

(c)

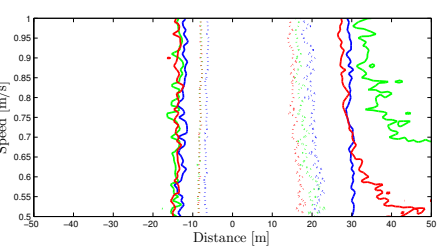

(d)

Fig. 5. Spawning results for the true tracks in Figure 1d. (a), (b) and (c) show the mean estimated cardinality for F1, F2 and F3, respectively. Dashed lines corresponds to cardinality 1.1, solid lines correspond to cardinality 1.9. (d) Contour plot of the estimated cardinality for F1 (blue), F2 (green), and F3 (red), respectively. Distance is computed as $x_{k}^{(1)}-x_{k}^{(2)}$, i.e. the difference in x-position, explaining why there are negative distances.

dominates the single target hypothesis easily when the targets are sufficiently separated. This happens earlier for F1 than F3.

The filter F2 always expects a single target. For obtaining the correct cardinality, it has to initiate/give birth to a new extended target. When the targets move/separate fast, the size of the single extended target predicted by the filter cannot catch up with the swiftly enlarged size of the measurement cluster (due to the target separation). Since the predicted target size remains small while the size of the measurement cluster becomes large, a new target is initialized/born easily under this estimate-measurement mismatch. Hence F2 can compensate the lack of spawning hypotheses by initiating a new extended target when the targets separate fast.

However, when the speeds of the targets are small (i.e. when the targets separate slowly), the predicted target size can easily match the overall measurement cluster size, and the incentive to initiate a new target is greatly reduced. Only when the targets are very far can F2 realize that a single elliptical target extent is too poor an explanation for the separated measurement clusters, and initiate a new target.

Hence when the targets separate slowly, new target initiation in F2 is delayed too much, and the lack of the spawning hypotheses becomes really critical.

3) Summary: To summarize, it is possible for the GGIWPHD filter to detect spawned targets when spawning hypotheses are not used, however it becomes increasingly difficult as the separation speed decreases. The GGIW-PHD filter with spawning hypotheses detects the spawned targets at the same distance, independent of the separation speed.

Further, used in the GGIW-PHD filter and run on the scenarios in this paper, the presented spawning method clearly outperforms the spawning method presented in [13].

\section{E. Cycle times}

Adding spawning hypotheses increases the number of GGIW components in the filter, and as a consequence the computational complexity increases. Conversely, using the combination functionality decreases the complexity. Mean cycle times for the scenarios in Figures 1a and $1 \mathrm{~b}$ are given in Tables III and IV, respectively. As expected, the mean cycle time increases when spawning hypotheses are used, and it decreases when target combination is used. Note that one should not compare the cycle times for the filter without spawning and the filter without combination, because, while the filters are identically implemented, they are run on different scenarios.
TABLE III

CyCle TIMES [s] FOR THE SCENARIO IN FIGURE 1A

\begin{tabular}{lccc} 
Filter & Mean & Median & St.dev. \\
\hline w comb & 0.11 & 0.06 & 0.17 \\
w/o comb & 0.25 & 0.12 & 0.34
\end{tabular}

TABLE IV

CyClE TIMES [s] FOR THE SCENARIO IN FigURE 1B

\begin{tabular}{lccc} 
Filter & Mean & Median & St.dev. \\
\hline F1 & 0.82 & 0.66 & 0.73 \\
F2 & 0.11 & 0.09 & 0.07 \\
F3 & 0.14 & 0.12 & 0.09
\end{tabular}

\section{CONCLuding Remarks}

This paper presented models for combination and spawning of extended targets modeled with random matrices. These models were then used in order to propose functions for multiple extended target tracking filters similar to those used in multiple point target tracking filters. Results show that with an appropriate combination criterion, two extended targets can be combined into one larger target when they are spatially close, and moving in the same direction, while at the same time taking care of their extensions. For spawning, the results show that by including spawning hypotheses the spawning events can be detected earlier than the case when the spawning hypotheses are not used. The results also show that the presented extended target spawning method outperforms earlier work on the topic.

The simulation study clearly shows that adding spawning hypotheses enables earlier detection of spawned targets, however this comes at the price of increased complexity. In the present implementation, spawning hypotheses are added in each time step for GGIW components with weights $w>0.5$. As an alternative, the measurement sets could be used to determine when it is appropriate to add spawning hypotheses.

The analysis in the paper is limited to the two target case. The results can be directly applicable to combination and spawning events with more than two targets, if the combination/spawning involves two (groups of) targets at a time. The analysis of target combination can be generalized to more than two targets combining at the same time with a considerable amount of work. The more challenging scenarios where more than two (groups of) targets are spawned from an extended target is left as an interesting topic of future work. The combination and spawning functions could also be tested 
on experimental data, e.g. from a laser range sensor, a radar sensor, or a camera.

\section{APPENDIX A}

PROPERTIES OF GAMMA DISTRIBUTED RANDOM VARIABLES

Let $\gamma_{1}$ and $\gamma_{2}$ be independent and Gamma distributed with equal inverse scale parameters,

$$
p\left(\gamma_{1}\right)=\mathcal{G} \mathcal{A M}\left(\gamma_{1} ; \alpha_{1}, \beta\right), \quad p\left(\gamma_{2}\right)=\mathcal{G} \mathcal{A M}\left(\gamma_{2} ; \alpha_{2}, \beta\right) .
$$

Then $\gamma=\gamma_{1}+\gamma_{2}$ is Gamma distributed [35]

$$
p(\gamma)=\mathcal{G} \mathcal{A M}\left(\gamma ; \alpha_{1}+\alpha_{2}, \beta\right)
$$

and $\bar{\gamma}_{1}=\frac{\gamma_{1}}{\gamma_{1}+\gamma_{2}}$ is Beta distributed [35]

$$
p\left(\bar{\gamma}_{1}\right)=\mathcal{B E}\left(\bar{\gamma}_{1} ; \alpha_{1}, \alpha_{2}\right) .
$$

Let $\bar{\gamma}_{2}=\frac{\gamma_{2}}{\gamma_{1}+\gamma_{2}}=1-\bar{\gamma}_{1}$. It follows immediately from the definition of the beta distribution that $\bar{\gamma}_{2}$ is beta distributed,

$$
p\left(\bar{\gamma}_{2}\right)=\mathcal{B E}\left(\bar{\gamma}_{2} ; \alpha_{2}, \alpha_{1}\right) .
$$

The first and second order moments of $\bar{\gamma}_{1}$ are

$$
\mathrm{E}\left[\bar{\gamma}_{1}\right]=\frac{\alpha_{1}}{\alpha_{1}+\alpha_{2}}, \quad \mathrm{E}\left[\bar{\gamma}_{1}^{2}\right]=\frac{\alpha_{1}\left(\alpha_{1}+1\right)}{\left(\alpha_{1}+\alpha_{2}\right)\left(\alpha_{1}+\alpha_{2}+1\right)},
$$

and consequently the expected value of $\bar{\gamma}_{1} \bar{\gamma}_{2}=\bar{\gamma}_{1}\left(1-\bar{\gamma}_{1}\right)$ is straightforward to compute.

\section{APPENDIX B \\ MATRIX PRODUCT OF SUM OF GAUSSIANS}

Let $\mathbf{x}_{1} \in \mathbb{R}^{n_{x}}$ and $\mathbf{x}_{2} \in \mathbb{R}^{n_{x}}$ be two independent Gaussian distributed random vectors with mean vectors $\mathbf{m}_{1} \in \mathbb{R}^{n_{x}}$ and $\mathbf{m}_{2} \in \mathbb{R}^{n_{x}}$ and covariance matrices $P_{1} \in \mathbb{S}_{+}^{n_{x}}$ and $P_{2} \in \mathbb{S}_{+}^{n_{x}}$, and let $H$ be a $d \times n_{x}$ matrix. Then the quantity $\mathbf{x}_{12}=H\left(\mathbf{x}_{1}-\mathbf{x}_{2}\right) \in \mathbb{R}^{d}$ is Gaussian distributed,

$$
\begin{aligned}
p\left(\mathbf{x}_{12}\right) & =\mathcal{N}\left(\mathbf{x}_{12} ; \mathbf{m}_{12}, P_{12}\right), \\
m_{12} & =H\left(\mathbf{m}_{1}-\mathbf{m}_{2}\right), \\
P_{12} & =H\left(P_{1}+P_{2}\right) H^{\mathrm{T}} .
\end{aligned}
$$

Let $M_{12}=\mathbf{m}_{12} \mathbf{m}_{12}^{\mathrm{T}}$. The expected value and covariance of the $d \times d$ matrix $X_{12}=\mathbf{x}_{12} \mathbf{x}_{12}^{\mathrm{T}}$ are given by [14]

$$
\begin{aligned}
\mathrm{E}\left[X_{12}\right]_{i j}= & P_{12, i j}+M_{12, i j}, \\
\operatorname{Cov}\left(X_{12}\right)_{i j k l}= & P_{12, i k} P_{12, j l}+P_{12, i l} P_{12, j k} \\
& +P_{12, j l} M_{12, i k}+P_{12, i l} M_{12, j k} \\
& +P_{12, j k} M_{12, i l}+P_{12, i k} M_{12, j l},
\end{aligned}
$$

where $\mathrm{E}\left[X_{12}\right]_{i j}$ is the expected value of the $i, j$ :th element of $X_{12}$, and $\operatorname{Cov}\left(X_{12}\right)_{i j k l}$ is the covariance of the $i, j$ :th and $k, l$ :th elements of $X_{12}$. The expected value (69a) is derived using the first and second order moments of $\mathbf{x}_{12}$, deriving the covariance $(69 \mathrm{~b})$ requires tedious calculations involving the first to fourth order moments of $\mathbf{x}_{12}$, see [14].

\section{APPENDIX C}

\section{MARGINAL DISTRIBUTION OF KINEMATICAL STATE}

The marginal distribution $p\left(\mathbf{x}_{k} \mid \mathbf{Z}^{k}\right)$ is a multivariate student-t distribution $[12]^{4}$, with expected value and covariance [12]

$$
\begin{aligned}
\mathrm{E}\left[\mathbf{x}_{k}\right] & =\mathbf{m}_{k \mid k}, \\
\mathrm{Cov}\left(\mathbf{x}_{k}\right) & =\frac{P_{k \mid k} \otimes V_{k \mid k}}{v_{k \mid k}+s-s d-2} \triangleq \hat{P}_{k \mid k},
\end{aligned}
$$

for $v_{k \mid k}>s d+2-s$. The multivariate student-t distribution can be approximated with a multivariate Gaussian distribution by analytical minimization of the KL-div. This gives the following marginal distribution,

$$
p\left(\mathbf{x}_{k} \mid \mathbf{Z}^{k}\right) \approx \mathcal{N}\left(\mathbf{x}_{k} ; \mathbf{m}_{k \mid k}, \hat{P}_{k \mid k}\right) .
$$

\section{APPENDIX D \\ EXPECTED VALUES}

\section{A. Gamma distributed random variables}

Let $\gamma_{1}$ and $\gamma_{2}$ be independent and gamma distributed

$$
\begin{aligned}
& p\left(\gamma_{1}\right)=\mathcal{G A M}\left(\gamma_{1} ; \alpha_{1}, \beta_{1}\right), \\
& p\left(\gamma_{2}\right)=\mathcal{G} \mathcal{A M}\left(\gamma_{2} ; \alpha_{2}, \beta_{2}\right),
\end{aligned}
$$

with $\beta_{1} \neq \beta_{2}$. The expected value of $\gamma=\gamma_{1}+\gamma_{2}$ is

$$
\mathrm{E}[\gamma]=\mathrm{E}\left[\gamma_{1}+\gamma_{2}\right]=\mathrm{E}\left[\gamma_{1}\right]+\mathrm{E}\left[\gamma_{2}\right]=\frac{\alpha_{1}}{\beta_{1}}+\frac{\alpha_{2}}{\beta_{2}} .
$$

Let $\bar{\gamma}_{1}=\frac{\gamma_{1}}{\gamma_{1}+\gamma_{2}}$. The expected value of $\log \bar{\gamma}_{1}$ can be rewritten as

$$
\begin{aligned}
\mathrm{E}\left[\log \bar{\gamma}_{1}\right] & =\mathrm{E}\left[\log \gamma_{1}\right]-\mathrm{E}\left[\log \left(\gamma_{1}+\gamma_{2}\right)\right] \\
& =\psi_{0}\left(\alpha_{1}\right)-\log \left(\beta_{1}\right)-\mathrm{E}\left[\log \left(\gamma_{1}+\gamma_{2}\right)\right] .
\end{aligned}
$$

There is no analytical solution to $\mathrm{E}\left[\log \left(\gamma_{1}+\gamma_{2}\right)\right]$, however it can be computed after Taylor expanding the function $\log \left(\gamma_{1}+\right.$ $\left.\gamma_{2}\right)$ around the point $\gamma_{1}^{0}=\mathrm{E}\left[\gamma_{1}\right]$ and $\gamma_{2}^{0}=\mathrm{E}\left[\gamma_{2}\right]$, which gives

$$
\mathrm{E}\left[\log \left(\gamma_{1}+\gamma_{2}\right)\right] \approx \log \left(\frac{\alpha_{1}}{\beta_{1}}+\frac{\alpha_{2}}{\beta_{2}}\right)-\frac{1}{2} \frac{\frac{\alpha_{1}}{\beta_{1}^{2}}+\frac{\alpha_{2}}{\beta_{2}^{2}}}{\left(\frac{\alpha_{1}}{\beta_{1}}+\frac{\alpha_{2}}{\beta_{2}}\right)^{2}} .
$$

\section{B. Inverse random matrix}

1) Inverse Wishart: Let $X$ be inverse Wishart distributed $p(X)=\mathcal{I W}_{d}(X ; v, V)$. Then $X^{-1}$ is Wishart distributed $p\left(X^{-1}\right)=\mathcal{W}_{d}\left(X^{-1} ; v-d-1, V^{-1}\right)$ [14, Theorem 3.4.1]. The expected value of $X^{-1}$ is [14, Theorem 3.3.15]

$$
\mathrm{E}\left[X^{-1}\right]=(v-d-1) V^{-1} .
$$

2) Wishart: Let $X$ be Wishart distributed $p(X)=$ $\mathcal{W}_{d}(X ; v, V)$. Then $X^{-1}$ is inverse Wishart distributed $p\left(X^{-1}\right)=\mathcal{I W}_{d}\left(X^{-1} ; v+d+1, V^{-1}\right) \quad[14$, Theorem 3.4.1]. The expected value of $X^{-1}$ is [14, Theorem 3.4.3]

$$
\mathrm{E}\left[X^{-1}\right]=\frac{V^{-1}}{(v-d-1)} \text {. }
$$

${ }^{4}$ See also [36]. 


\section{Log-determinant of random matrix}

Let $y$ be a uni-variate random variable. The moment generating function for $y$ is defined as $\mu_{y}(s) \triangleq \mathrm{E}\left[e^{s y}\right]$, and the expected value of $y$ is given in terms of $\mu_{y}(s)$ as

$$
\mathrm{E}[y]=\left.\frac{\mathrm{d} \mu_{y}(s)}{\mathrm{d} s}\right|_{s=0} .
$$

1) Inverse Wishart: Let $y=\log |X|$, where $p(X)=$ $\mathcal{I W}_{d}(X ; v, V)$. The moment generating function of $y$ is

$$
\begin{aligned}
\mu_{y}(s) & =\mathrm{E}\left[|X|^{s}\right]=\int|X|^{s} p(X) \mathrm{d} X \\
& =\frac{\Gamma_{d}\left(\frac{v-d-1}{2}-s\right)}{\Gamma_{d}\left(\frac{v-d-1}{2}\right)}\left(\frac{|V|}{2^{d}}\right)^{s} .
\end{aligned}
$$

By [14, Theorem 1.4.1], the logarithm of $\Gamma_{d}(\cdot)$ can be expressed as

$$
\log \Gamma_{d}(a)=\frac{1}{4} d(d-1) \log \pi+\sum_{i=1}^{d} \log \Gamma\left(a-\frac{i-1}{2}\right)
$$

The expected value of $y$ is

$$
\begin{aligned}
\mathrm{E}[y] & =\mathrm{E}[\log |X|] \\
& =\left.\frac{\mathrm{d}}{\mathrm{d} s}\left(\frac{\Gamma_{d}\left(\frac{v-d-1}{2}-s\right)}{\Gamma_{d}\left(\frac{v-d-1}{2}\right)}\left(\frac{|V|}{2^{d}}\right)^{s}\right)\right|_{s=0} \\
& =\log |V|-d \log 2-\sum_{j=1}^{d} \psi_{0}\left(\frac{v-d-j}{2}\right) .
\end{aligned}
$$

2) Wishart: Let $y=\log |X|$, where $p(X)=$ $\mathcal{W}_{d}(X ; v, V)$. Analogously to the derivation above, the expected value of $y$ is

$$
\begin{aligned}
\mathrm{E}[y] & =\mathrm{E}[\log |X|] \\
& =\log |V|+d \log 2+\sum_{j=1}^{d} \psi_{0}\left(\frac{v-j+1}{2}\right) .
\end{aligned}
$$

\section{APPENDIX E}

\section{PROOF OF THEOREM 1}

Proof: We have $q(\cdot)$ given by

$$
\begin{aligned}
& q(\gamma) \triangleq \underset{q}{\arg \min } \mathrm{KL}(p \| q) \\
&=\underset{q}{\arg \max } \int p(\gamma) \log q(\gamma) \mathrm{d} \gamma \\
&=\underset{q}{\arg \max }(\alpha \log \beta-\log \Gamma(\alpha) \\
&\left.\quad+(\alpha-1) \mathrm{E}_{p}[\log (\gamma)]-\beta \mathrm{E}_{p}[\gamma]\right) .
\end{aligned}
$$

Differentiating the objective function with respect to $\beta$, setting the result equal to zero, and solving for $\beta$, gives

$$
\beta=\frac{\alpha}{\mathrm{E}_{p}[\gamma]} .
$$

Differentiating the objective function with respect to $\alpha$, setting the result equal to zero, and inserting $\beta$ given in (84), gives

$$
\log (\alpha)-\psi_{0}(\alpha)+\mathrm{E}_{p}[\log (\gamma)]-\log \left(\mathrm{E}_{p}[\gamma]\right)=0 .
$$

\section{APPENDIX F}

PROOF OF THEOREM 2

Proof: We have $q(\cdot)$ given by

$$
\begin{aligned}
q(\gamma) \triangleq & \underset{q}{\arg \min } \mathrm{KL}(p \| q) \\
= & \underset{q}{\arg \max } \int p\left(\bar{\gamma}_{1}\right) \log q\left(\bar{\gamma}_{1}\right) \mathrm{d} \bar{\gamma}_{1} \\
= & \underset{q}{\arg \max }(\log \Gamma(a+b)-\log \Gamma(a)-\log \Gamma(b) \\
& \left.+(a-1) \mathrm{E}\left[\log \left(\bar{\gamma}_{1}\right)\right]+(b-1) \mathrm{E}\left[\log \left(1-\bar{\gamma}_{1}\right)\right]\right) .
\end{aligned}
$$

Differentiating the objective function with respect to $a$, and setting the result equal to zero gives

$$
\psi_{0}(a+b)-\psi_{0}(a)+\mathrm{E}\left[\log \left(\bar{\gamma}_{1}\right)\right]=0 .
$$

Differentiating the objective function with respect to $b$, and setting the result equal to zero gives

$$
\psi_{0}(a+b)-\psi_{0}(b)+\mathrm{E}\left[\log \left(\bar{\gamma}_{2}\right)\right]=0,
$$

where $\bar{\gamma}_{2}=\frac{\gamma_{2}}{\gamma_{1}+\gamma_{2}}=1-\bar{\gamma}_{1}$.

\section{APPENDIX G}

\section{PRoOF OF THEOREM 3}

Proof: We have $q(\cdot)$ given as

$$
\begin{aligned}
q(X) \triangleq & \underset{q}{\arg \min } \mathrm{KL}(p \| q) \\
= & \underset{q}{\arg \max } \int p(X) \log (q(X)) \mathrm{d} X \\
= & \underset{q}{\arg \max }\left[\frac{1}{2}(v-d-1) \mathrm{E}_{p}[\log |X|]\right. \\
& -\frac{1}{2} \operatorname{Tr}\left(V^{-1} \mathrm{E}_{p}[X]\right)-\frac{1}{2} v d \log (2) \\
& \left.-\Gamma_{d}(v / 2)-\frac{1}{2} v \log |V|\right]
\end{aligned}
$$

Taking the derivative of the objective function with respect to $V$, equating the result to zero, and solving for $V$, we get

$$
V=\frac{1}{v} \mathrm{E}_{p}[X]
$$

Now, we take the derivative of the objective function with respect to $v$, equate the result to zero, and insert the $V$ in (90), to obtain

$$
\begin{aligned}
& \sum_{i=1}^{d} \psi_{0}((v-i+1) / 2)+d \log (v / 2) \\
& -\mathrm{E}_{p}[\log |X|]+\log \left|\mathrm{E}_{p}[X]\right|=0 .
\end{aligned}
$$




\section{APPENDIX H}

\section{PROOF OF THEOREM 4}

Proof: We have $q(\cdot)$ given as

$$
\begin{aligned}
q(X) \triangleq & \underset{q}{\arg \min } \mathrm{KL}(p \| q) \\
= & \underset{q}{\arg \max } \int p(X) \log (q(X)) \mathrm{d} X \\
= & \underset{q}{\arg \max }\left[\frac{1}{2}(v-d-1) \log |V|\right. \\
& -\frac{1}{2} \operatorname{Tr}\left(V \mathrm{E}_{p}\left(X^{-1}\right)\right)-\frac{1}{2}(v-d-1) d \log (2) \\
& \left.-\log \Gamma_{d}((v-d-1) / 2)-\frac{1}{2} v \mathrm{E}_{p}(\log |X|)\right]
\end{aligned}
$$

Taking the derivative of the objective function with respect to $V$, equating the result to zero, and solving for $V$, we get

$$
V=(v-d-1)\left[\mathrm{E}_{p}\left(X^{-1}\right)\right]^{-1}
$$

Now, we take the derivative of the objective function with respect to $v$, equate the result to zero, and insert the $V$ in (93), to obtain

$$
\begin{array}{r}
-\sum_{i=1}^{d} \psi_{0}((v-d-i) / 2)+d \log ((v-d-1) / 2) \\
-\mathrm{E}_{p}(\log |X|)-\log \left|\mathrm{E}_{p}\left(X^{-1}\right)\right|=0 .
\end{array}
$$

\section{ACKNOWLEDGMENT}

The authors would like to thank the Linnaeus research environment CADICS and the frame project grant Extended Target Tracking (621-2010-4301), both funded by the Swedish Research Council, and the project Collaborative Unmanned Aircraft Systems (CUAS), funded by the Swedish Foundation for Strategic Research (SSF), for financial support.

\section{REFERENCES}

[1] Y. Bar-Shalom and T. E. Fortmann, Tracking and data association, ser. Mathematics in Science and Engineering. San Diego, CA, USA: Academic Press Professional, Inc., 1987, vol. 179.

[2] Y. Bar-Shalom, Multitarget-multisensor tracking: applications and advances. Artech House, 1992, vol. II.

[3] R. Mahler, Statistical Multisource-Multitarget Information Fusion. Norwood, MA, USA: Artech House, 2007.

[4] B. Lau, K. O. Arras, and W. Burgard, "Multi-model hypothesis group tracking and group size estimation," International Journal of Social Robotics, vol. 2, no. 1, pp. 19-30, Mar. 2010.

[5] D. J. Salmond and M. C. Parr, "Track maintenance using measurements of target extent," IEE Proceedings - Radar, Sonar and Navigation, vol. 150, no. 6, pp. 389-395, Dec. 2003.

[6] M. Baum and U. D. Hanebeck, "Random hypersurface models for extended object tracking," in IEEE International Symposium on Signal Processing and Information Technology (ISSPIT), Ajman, United Arab Emirates, Dec. 2009, pp. 178-183.

[7] M. Baum, B. Noack, and U. D. Hanebeck, "Extended Object and Group Tracking with Elliptic Random Hypersurface Models," in Proceedings of the International Conference on Information Fusion, Edinburgh, UK, Jul. 2010.

[8] M. Baum and U. D. Hanebeck, "Shape Tracking of Extended Objects and Group Targets with Star-Convex RHMs," in Proceedings of the International Conference on Information Fusion, Chicago, IL, USA, Jul. 2011, pp. 338-345.
[9] K. Granström, C. Lundquist, and U. Orguner, "Tracking Rectangular and Elliptical Extended Targets Using Laser Measurements," in Proceedings of the International Conference on Information Fusion, Chicago, IL, USA, Jul. 2011, pp. 592-599.

[10] C. Lundquist, K. Granström, and U. Orguner, "Estimating the Shape of Targets with a PHD Filter," in Proceedings of the International Conference on Information Fusion, Chicago, IL, USA, Jul. 2011, pp. 49-56.

[11] H. Zhu, C. Han, and C. Li, "An extended target tracking method with random finite set observations," in Proceedings of the International Conference on Information Fusion, Chicago, IL, USA, Jul. 2011, pp 73-78.

[12] J. W. Koch, "Bayesian approach to extended object and cluster tracking using random matrices," IEEE Transactions on Aerospace and Electronic Systems, vol. 44, no. 3, pp. 1042-1059, Jul. 2008.

[13] F. Lian, C.-Z. Han, W.-F. Liu, X.-X. Yan, and H.-Y. Zhou, "Sequential Monte Carlo implementation and state extraction of the group probability hypothesis density filter for partly unresolvable group targets-tracking problem," IET Radar, Sonar and Navigation, vol. 4, no. 5, pp. 685-702, Oct. 2010.

[14] A. K. Gupta and D. K. Nagar, Matrix variate distributions, ser. Chapman \& Hall/CRC monographs and surveys in pure and applied mathematics. Chapman \& Hall, 2000.

[15] M. Feldmann, D. Fränken, and J. W. Koch, "Tracking of extended objects and group targets using random matrices," IEEE Transactions on Signal Processing, vol. 59, no. 4, pp. 1409-1420, Apr. 2011.

[16] K. Granström and U. Orguner, "Estimation and Maintenance of Measurement Rates for Multiple Extended Target Tracking," in Proceedings of the International Conference on Information Fusion, Singapore, Jul. 2012, pp. 2170-2176.

[17] A. Gelman, J. B. Carlin, H. S. Stern, and D. B. Rubin, Bayesian Data Analysis, ser. Texts in Statistical Science. Chapman \& Hall/CRC, 2004.

[18] S. Kullback and R. A. Leibler, "On information and sufficiency," The Annals of Mathematical Statistics, vol. 22, no. 1, pp. 79-86, Mar. 1951.

[19] J. L. Williams and P. S. Maybeck, "Cost-Function-Based Gaussian Mixture Reduction for Target Tracking," in Proceedings of the International Conference on Information Fusion, Cairns, Queensland, Australia, Jul. 2003.

[20] A. R. Runnalls, "Kullback-Leibler approach to Gaussian mixture reduction," IEEE Transactions on Aerospace and Electronic Systems, vol. 43, no. 3, pp. 989-999, Jul. 2007.

[21] D. Schieferdecker and M. F. Huber, "Gaussian Mixture Reduction via Clustering," in Proceedings of the International Conference on Information Fusion, Seattle, WA, USA, Jul. 2009.

[22] J. Stoer and R. Bulirsch, Introduction to Numerical Analysis, 2nd ed New York: Springer-Verlag, 1993.

[23] S. Boyd and L. Vandenberghe, Convex Optimization. New York, NY, USA: Cambridge University Press, 2004.

[24] J. Löfberg, "Yalmip : A toolbox for modeling and optimization in MATLAB," in Proceedings of the CACSD Conference, Taipei, Taiwan, 2004. [Online]. Available: http://users.isy.liu.se/johanl/yalmip

[25] —_ "Automatic robust convex programming," Optimization methods and software, vol. 27, no. 1, pp. 115-129, 2012.

[26] M. Grant and S. Boyd, "CVX: Matlab software for disciplined convex programming, version 1.21,”..././cvx, Apr. 2011.

[27] — "Graph implementations for nonsmooth convex programs," in Recent Advances in Learning and Control, ser. Lecture Notes in Control and Information Sciences, V. Blondel, S. Boyd, and H. Kimura, Eds. Springer-Verlag Limited, 2008, pp. 95-110, http://stanford.edu/ boyd/ graph_dcp.html.

[28] D. E. Clark and S. J. Godsill, "Group target tracking with the Gaussian mixture probability hypothesis density filter," in International Conference on Intelligent Sensors, Sensor Networks and Information (ISSNIP), Melbourne, Australia, Dec. 2007, pp. 149-154.

[29] K. Granström and U. Orguner, "A PHD filter for tracking multiple extended targets using random matrices," IEEE Transactions on Signal Processing, vol. 60, no. 11, pp. 5657-5671, Nov. 2012.

[30] K. Gilholm, S. Godsill, S. Maskell, and D. Salmond, "Poisson models for extended target and group tracking," in Proceedings of Signal and Data Processing of Small Targets, vol. 5913. San Diego, CA, USA: SPIE, Aug. 2005, pp. 230-241.

[31] K. Granström and U. Orguner, "Implementation of the GIW-PHD filter," Department of Electrical Engineering, Linköping University, SE-581 83 Linköping, Sweden, Tech. Rep. LiTH-ISY-R-3046, Mar. 2012. [Online]. Available: http://www.control.isy.liu.se/publications/doc?id=2508 
[32] R. Mahler, "PHD filters for nonstandard targets, I: Extended targets," in Proceedings of the International Conference on Information Fusion, Seattle, WA, USA, Jul. 2009, pp. 915-921.

[33] B.-N. Vo and W.-K. Ma, "The Gaussian mixture probability hypothesis density filter," IEEE Transactions on Signal Processing, vol. 54, no. 11, pp. 4091-4104, Nov. 2006.

[34] D. Schuhmacher, B.-T. Vo, and B.-N. Vo, "A consistent metric for performance evaluation of multi-object filters," IEEE Transactions on Signal Processing, vol. 56, no. 8, pp. 3447-3457, Aug. 2008.

[35] M. V. Jambunathan, "On information and sufficiency," The Annals of Mathematical Statistics, vol. 25, no. 2, pp. 401-405, Jun. 1954.

[36] M. Feldmann and W. Koch, "Comments on "Bayesian Approach to Extended Object and Cluster Tracking using Random Matrices"," IEEE Transactions on Aerospace and Electronic Systems, vol. 48, no. 2, pp. 1687-1693, Apr. 2012.

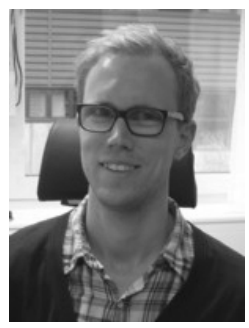

Karl Granström received the M.Sc. degree in Applied Physics and Electrical Engineering in 2008, and the Tech.Lic. degree in Automatic Control in 2011, both from Linköping University, Linköping, Sweden. At present he is working towards a Ph.D. degree at the Division of Automatic Control at the Department of Electrical Engineering, Linköping University.

His research interests include sensor fusion, target tracking, mapping and localization, especially for mobile robots.

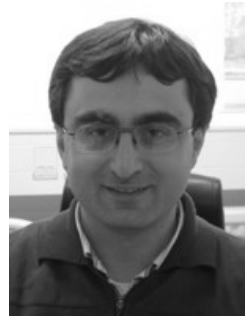

Umut Orguner is an Assistant Professor at Department of Electrical \& Electronics Engineering of Middle East Technical University, Ankara, Turkey. $\mathrm{He}$ got his B.S., M.S. and Ph.D. degrees all in Electrical Engineering from the same department in 1999, 2002 and 2006 respectively. He held a postdoctoral position between 2007 and 2009 at the Division of Automatic Control at the Department of Electrical Engineering of Linköping University, Linköping, Sweden. Between 2009 and 2012 he was an Assistant Professor at the Division of Automatic Control at the Department of Electrical Engineering of Linköping University, Linköping, Sweden.

His research interests include estimation theory, multiple-model estimation, target tracking, and information fusion. 